\title{
$\alpha$-(Imino)pyridyldifluoroethyl Phosphonates: Novel Promising Building Blocks in Synthesis of Biorelevant Aminophosphonic Acids Derivatives
}

\author{
Oksana M. Shavrina ${ }^{1}$, Lyudmyla V. Bezgubenko ${ }^{1}$, Andrii V. Bezdudny ${ }^{1}$, Petro P. Onys'ko ${ }^{1, * \mathbb{D}}$ \\ and Yuliya V. Rassukana ${ }^{1,2}$
}

1 Institute of Organic Chemistry, National Academy of Sciences of Ukraine, 5 Murmans'ka Str., 02660 Kyiv, Ukraine; petruk94oksana@ukr.net (O.M.S.); bezgubenko@ukr.net (L.V.B.); bezdudny@ioch.kiev.ua (A.V.B.); juravi@ioch.kiev.ua (Y.V.R.)

2 Department of Organic Chemistry, National Technical University of Ukraine "Kyiv Polytechnic Institute", 37, Prospect Peremogy, 03056 Kyiv, Ukraine

* Correspondence: onysko@ioch.kiev.ua

\section{check for}

updates

Citation: Shavrina, O.M.;

Bezgubenko, L.V.; Bezdudny, A.V.;

Onys'ko, P.P.; Rassukana, Y.V.

$\alpha$-(Imino)pyridyldifluoroethyl

Phosphonates: Novel Promising

Building Blocks in Synthesis of

Biorelevant Aminophosphonic Acids

Derivatives. Organics 2021, 2, 72-83.

https://doi.org/10.3390/org2020007

Academic Editor: Tomasz

K. Olszewski

Received: 7 March 2021

Accepted: 21 April 2021

Published: 23 April 2021

Publisher's Note: MDPI stays neutral with regard to jurisdictional claims in published maps and institutional affiliations.

Copyright: (c) 2021 by the authors. Licensee MDPI, Basel, Switzerland. This article is an open access article distributed under the terms and conditions of the Creative Commons Attribution (CC BY) license (https:/ / creativecommons.org/licenses/by/ $4.0 /)$.

\begin{abstract}
A convenient synthetic approach to previously unknown $\mathrm{NH}$-iminophosphonates bearing 2-, 3-, and 4-pyridyldifluoromethyl groups at the imine carbon atom was developed. The synthetic potential of these novel building blocks was demonstrated by their conversion into highly functionalized acyclic and heterocyclic aminophosphonates and phosphonic acids combining in their structure biorelevant aminophosphonic fragment, difluoromethyl group, and pyridyl, piperidyl, thiazolidin-4-one, or thiazidinan-4-one heterocyclic moieties in a single molecular platform.
\end{abstract}

Keywords: iminophosphonates; aminophosphonates; NH-imines; nitriles; difluoromethyl; pyridine; piperidine; E-Z isomerism

\section{Introduction}

$\alpha$-Aminophosphonates are phosphorus analogs of amino acids in which the planar carboxylic group is replaced with a tetrahedral bioisosteric phosphonic unit. They exhibit a wide range of biological activity and play a significant role in the development of antibiotics, antiviral, antihypertensive, antitumor agents and other bioactive substances [1-5]. Of particular interest are fluorinated aminophosphonates. Modification of organic molecule with fluorine has become almost a standard tool in modern drug design. It is generally accepted that introduction of a fluorine containing group may improve the pharmacodynamic and the pharmacokinetic profiles of the compound by concomitant alteration of its electronic, lipohilic, and steric characteristics as well as its metabolic stability [6-9].

$\alpha$-Iminophosphonates, i.e., the compounds bearing $(\mathrm{RO})_{2} \mathrm{P}(\mathrm{O}) \mathrm{C}=\mathrm{N}$-fragment, are valuable precursors of $\alpha$-aminophosphonates. Unprotected $\mathrm{NH}$-iminophosphonates are especially promising in this respect. They show enhanced reactivity and allow straightforward preparation of aminophosphonates with a free $\mathrm{NH}_{2}$ group avoiding tedious $\mathrm{N}$-deprotection step. Recently we have prepared the first representatives of $\mathrm{NH}$-iminophosphonates and demonstrated their potential for the introduction of pharmacophore $\alpha$-fluoroalkylated aminophosphonic unit into various structures.

NH-Iminophosphonates incorporating heterocyclic residue were unknown so far. In the present work we report the synthesis of $\mathrm{NH}$-iminophosphonates bearing $\alpha-, \beta-$, or $\gamma$-pyridyldifluoromethyl group at the imine carbon atom and their use for the preparation of aminophosphonic acids derivatives bearing difluoromethyl group and heterocyclic residue. Note, that compounds with 2-pyridyldifluoromethyl fragment were used for the development of effective thrombin and trypsin inhibitors [10] and highly selective antifungal agents [11]. The presence of a pyridine ring offers additional synthetic possibilities connected with the hydrogenation of heterocyclic ring and preparation of compounds 
containing aminophosphonic unit, difluoromethyl group and piperidine fragment in the same molecule. It is worth of noting that according to [12] piperidine and pyridine are most frequent nitrogen heterocycles in U.S. FDA approved drugs.

\section{Results and Discussion}

Analysis of the literature data shows that hydrophosphoryl compounds can react with nitriles by different schemes. Thus, reaction of dialkylphosphites with alkyl, cycloalkyl, aryl, or pyridyl nitriles in the presence of acids [13,14] or under free radical conditions [15] leads to aminobisphosphonates $\mathbf{I}$, as the main products, formed in low to moderate yields (Scheme 1). At the same time, under basic conditions benzonitrile reacts with dialkyl or diphenyl phosphites to form phosphorylamino phosphonates II [16]. Recently we have shown that highly electrophilic polyfluoroalkylated nitriles in the reactions with dialkyl or diphenyl phosphite afford first representatives of NH-iminophosphonates III [17,18]. At the same time, mixed perfluoro(chloro) acetonitriles react with $(\mathrm{RO}){ }_{2} \mathrm{POH}(\mathrm{R}=\mathrm{Et}, \mathrm{Ph})$ by two competitive routes: addition to the $\mathrm{C} \equiv \mathrm{N}$ bond affording the respective $\mathrm{N}$-unprotected iminophosphonates III, or reductive dehalogenation leading to chloro(fluoro) acetonitriles IV and the respective halogenophosphates $\mathbf{V}$ [19].

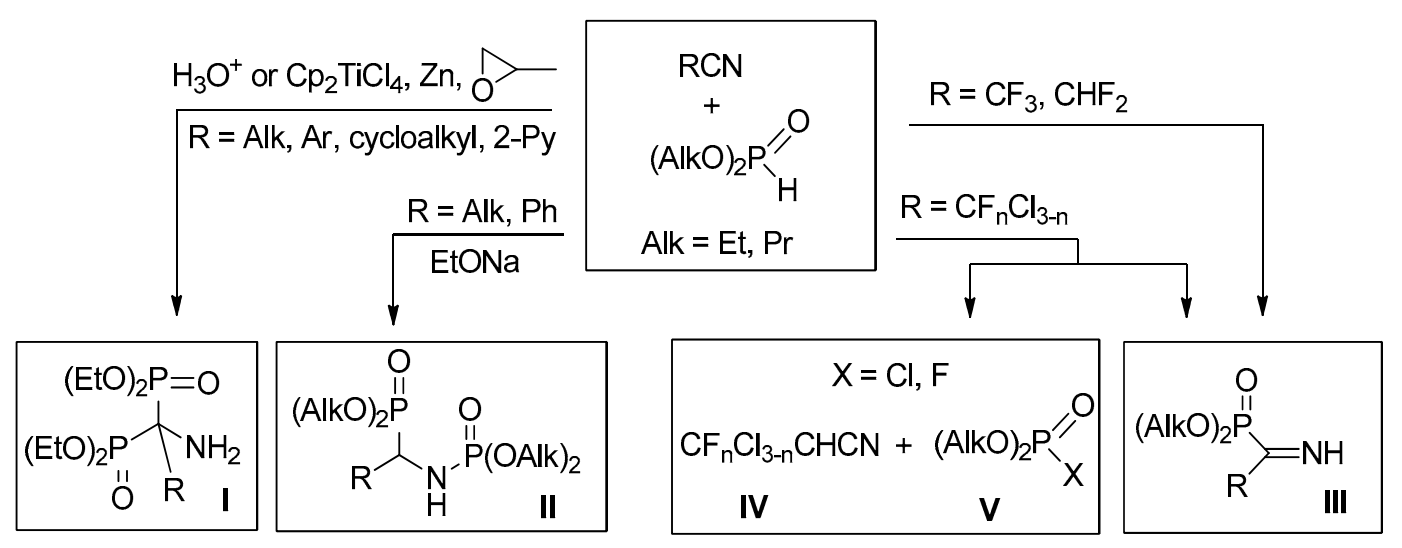

Scheme 1. Reactions of nitriles with hydrophosphoryl compounds.

Thus, the results of reactions of hydrophosphoryl compounds with nitriles depend on reactants structure and conditions.

We have found that all three isomeric pyridyldifluoroacetonitriles 1a-c react with diethyl phosphite under mild conditions $\left(20 \mathrm{~mol} . \% \mathrm{Et}_{3} \mathrm{~N}\right.$, r.t.) to afford respective NHiminophosphonates $\mathbf{2 a - c}$ (Scheme 2). It should be noted that 2-pyridyl nitrile 1a reacts much more slowly than the 3 - and 4 -isomers $\mathbf{1 b}, \mathbf{c}$ : under the same conditions the reaction is completed within 120 and $12 \mathrm{~h}$, respectively. Similar differences have previously been noted for $\mathrm{pKa}$ values of the isomeric pyridineacetic acids [20] which may be associated with the specific effect of the $\alpha$-nitrogen atom.

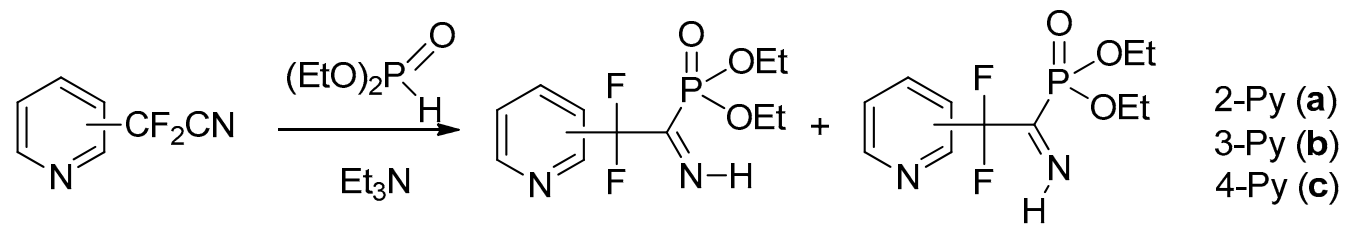

(E-) 2a-c

Scheme 2. Synthesis of pyridyldifluoromethylated NH-iminophosphonates.

Formation of the P-C=N-H fragment is confirmed by ${ }^{13} \mathrm{C}$ NMR spectra of compounds 2, in which the imine $C$-atom signal $\left(\delta_{C} 171-173 \mathrm{ppm}\right)$ with a large direct C-P coupling 
constant $\left({ }^{1} J_{\mathrm{CP}} 153-154 \mathrm{~Hz}\right.$ for Z-izomers $\mathbf{2 a}-\mathbf{c}$; $210 \mathrm{~Hz}$ for E-isomer 2a) was identified, and ${ }^{1} \mathrm{H},{ }^{31} \mathrm{P}$ NMR spectra showing characteristic spin-spin interaction of the $\mathrm{N}-\mathrm{H}$ proton and phosphorus atom $\left({ }^{3} J_{\mathrm{P}-\mathrm{C}-\mathrm{N}-\mathrm{H}} 40-62 \mathrm{~Hz}\right)$.

Iminophosphonates $\mathbf{2 a - c}$ are formed as an equilibrium mixture of $Z / E$ isomers and, similarly to other fluoroalkylated $\mathrm{NH}$-iminophosphonates, a more sterically hindered $Z$ configuration is preferred. We have shown previously that substituent at the $C=N$ bond of iminophosphonates markedly affect $E / Z$ isomeric ratio: C-trifluoromethylated iminophosphonates exist preferentially in $Z$ configuration, whereas for their $C$-arylated analogs $E$-configuration prevails [21,22]. Table 1 shows the values of equilibrium $Z / E$ ratios and phosphorus chemical shifts for pyridyldifluoromethylphosphonates $\mathbf{2 a}-\mathbf{c}$ and some other related iminophosphonates. It is seen that ${ }^{31} \mathrm{P} N M R$ data and $Z / E$ ratios for iminophosphonates $\mathbf{2} \mathbf{b}, \mathbf{c}$ are very close and markedly differ from those for isomer $\mathbf{2 a}$. It is very interesting to note from analysis of Table 1 that imines $\mathbf{2} \mathbf{b}, \mathbf{c}$ bearing more strong electron withdrawing 3- and 4-pyridyl substituent $\left(\sigma_{p} 0.25\right.$ and 0.44 , respectively [23] are in this respect more similar to trifluoromethylated analog 3 , whereas isomer $\mathbf{2 a}$ with less electron withdrawing 2-pyridyl group $\left(\sigma_{\mathrm{p}} 0.17\right)$ is more like benzimidoyl phosphonates 4,5 .

Table 1. $Z / E$ isomeric ratio and selected ${ }^{13} \mathrm{C},{ }^{31} \mathrm{P}$ NMR data of the compounds 2 and some related iminophosphonates.

\begin{tabular}{|c|c|c|c|c|c|}
\hline \multirow[t]{2}{*}{ Compound } & \multirow{2}{*}{$\begin{array}{c}\mathrm{Z/E} \\
\left(\mathrm{CDCl}_{3}\right)\end{array}$} & \multicolumn{2}{|c|}{$\delta_{P}\left({ }^{3} J_{P-C=N-H}\right)$} & \multicolumn{2}{|c|}{$\delta_{C=N}\left({ }^{1} J_{C P}\right)$} \\
\hline & & Z & $E$ & Z & $E$ \\
\hline $2 a$ & $2: 1$ & $5.9(39.9)$ & $6.6(61.6)$ & $173.2(153)$ & $170.8(210)$ \\
\hline $2 b$ & $6: 1$ & $1.2(39.6)$ & $3.0(61.2)$ & $173(154)$ & - \\
\hline $2 c$ & $7: 1$ & $0(39.5)$ & $1.8(62.2)$ & $172.9(154)$ & - \\
\hline $\mathrm{CF}_{3} \mathrm{CP}(\mathrm{O})(\mathrm{OEt})_{2}=\mathrm{NH}(3)$ & $10: 1$ & $-1.4(37.2)$ & $0.7(58.2)$ & $166.8(163.5)$ & - \\
\hline $\mathrm{PhCP}(\mathrm{O})(\mathrm{OEt})_{2}=\mathrm{NH}(4)$ & $3: 1$ & $3.9(46)$ & $6.5(75)$ & - & - \\
\hline $\mathrm{PhCP}(\mathrm{O})(\mathrm{OEt})_{2}=\mathrm{NMe}(5)$ & $1: 17$ & 2.3 & 6.9 & - & $169.3(220)$ \\
\hline
\end{tabular}

Identification of $Z, E$-isomers $\mathbf{2} \mathbf{a}-\mathbf{c}$ is based mainly on the significant difference in coupling constants of the N-H proton and phosphorus atom ( ${ }^{3} J_{\mathrm{P}-\mathrm{C}-\mathrm{N}-\mathrm{H}} \sim 40$ and $\sim 61-62 \mathrm{~Hz}$, respectively) and high-field shift of phosphorus signals of $Z$-isomers in ${ }^{31} \mathrm{P}$ NMR spectra, previously noted for other $\alpha$-iminophosphonates [17,18,21,22].

Iminophosphonates 2 contain a polarized $\mathrm{C}=\mathrm{N}$ bond activated by the presence of electron-withdrawing group at the imine carbon atom. Thus, imines $2 \mathbf{a}-\mathbf{c}$ readily add methanol across the $\mathrm{C}=\mathrm{N}$ bond to afford adduct $\mathbf{6 a}-\mathbf{c}$ (Scheme 3). The reactivity decreases in the order $\mathbf{2 a}>\mathbf{2 b}$,c: after $8 \mathrm{~h}$ the conversion is $100 \%, 44 \%$, and $46 \%$, respectively. The complete conversion of $\mathbf{2} \mathbf{b}, \mathbf{c}$ proceeds within 2 days.

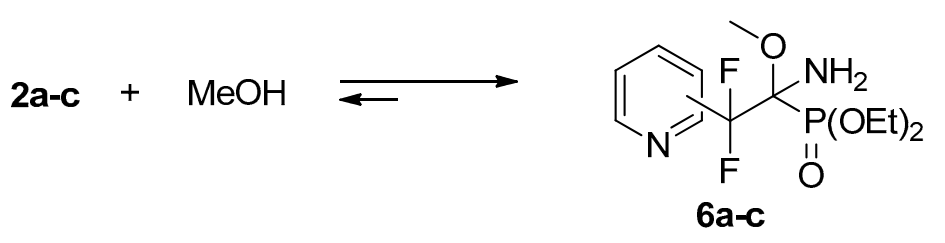

Scheme 3. Reversible reaction of iminophosphonates 2 with methanol.

Similar to trifluoromethylated analogs $[17,18,21]$, compounds $6 \mathbf{a}-\mathbf{c}$ in $\mathrm{CDCl}_{3}$ solution partially $(\sim 15-20 \%)$ dissociate to the starting compounds. This property of NH-imine adducts was successfully explored for the improvement of the enantiomeric excess in catalytic asymmetric reduction [24].

It is very interesting to note, that addition of methanol to iminophosphonates $\mathbf{2}$ is accompanied by the change in the $Z / E$ ratio of the starting compounds and $Z$ - $E$ equilibrium is established very quickly. Thus, immediately after dissolution of imines $\mathbf{2 a}-\mathbf{c}$ in methanol, $\mathrm{Z} / \mathrm{E}$ ratios of imines $\mathbf{2 a -}-\mathbf{c}$, according to ${ }^{31} \mathrm{P},{ }^{19} \mathrm{~F}$ NMR data, are 1:1, 2.5:1, and 2:1, respectively. 
Evaporation of methanol and dissolution in deuterochloroform leads to recovery of $Z$ / $E$ isomeric ratios, presented in Table 1, i.e., 2:1, 6:1, and 7:1, respectively. Similar increase of $E$-isomer content on going from aprotic to protic solvent was shown recently for the sodium salts of $\mathrm{N}$-methyliminophosphonates, bearing aryl or hetaryl substituents at the imine carbon atom [22].

Synthesis of biorelevant pirydyldifluoroemethylated aminophosphonates and aminophosphonic acids. We have found that selective reduction of the $\mathrm{C}=\mathrm{N}$ bond in iminophosphonates $\mathbf{2 a - c}$ with borane-dimethylsulfide (BMS) proceeds under mild conditions to give aminophosphonates 7a-c incorporating pyridyldifluoromethyl residue (Scheme 4). Free aminophosphonates $7 \mathbf{a}-\mathbf{c}$ are not very stable (the stability decreases in the order $7 \mathrm{c}>7 \mathbf{b}>\mathbf{7 a}$ ) and should be stored at the reduced temperature in refrigerator.

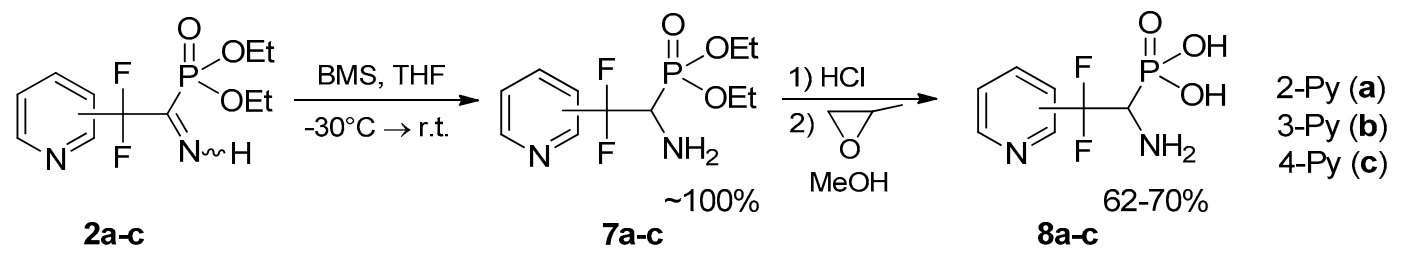

Scheme 4. Selective reduction of the $\mathrm{C}=\mathrm{N}$ bond in the iminophosphonates $\mathbf{2 a}-\mathbf{c}$.

Preparation of heterocycle-containing aminophosphonic acids from their esters are often complicated by side processes. It was found that hydrolysis of aminophosphonates $7 \mathrm{a}-\mathrm{c}$ with conc. $\mathrm{HCl}$ proceeds cleanly and affords the first representatives of fluoroalkylated aminophosphonic acids $\mathbf{8 a}-\mathbf{c}$, incorporating heterocyclic moiety in the $\beta$-position.

The possibility of reducing the pyridine ring was studied using compound $7 \mathrm{c}$ as the most stable pyridyl substituted aminophosphonate. It was found that catalytic hydrogenation of $7 \mathrm{c}$ leads to complete reduction of pyridine ring to afford 4-piperidyl substituted aminophosphonate 9 (Scheme 5).<smiles>CCOC(=O)C(F)(F)C(N)(c1ccncc1)C(N)P(=O)(OCC)OCC</smiles>

Scheme 5. Catalytic hydrogenation of the aminophosphonate 7c.

The latter should be stored at a reduced temperature, since under ordinary conditions it gradually decomposes, most likely undergoing O-N transfer of the ethyl group. More stable free aminophosphonic acid $\mathbf{1 0}$ was obtained by hydrolysis of $\mathbf{9}$ followed by treatment with propylene oxide.

Cyclocondensation with mercaptocarboxylic acids. Highly polarized azomethyne bond in the imines 2 can be readily functionalized by the reactions with the bifunctional compounds. Thus, cyclocondensation of iminophosphonates $\mathbf{2 a - c}$ with thioglycolic or 3-mercaptopropionic acid leads to highly functionalized thiazolidin-4-ones 11a-c or thiazidinan-4-ones 12a-c, respectively (Scheme 6). Obviously, the reaction proceeds via primary nucleophilic addition to the $\mathrm{C}=\mathrm{N}$ bond followed by intramolecular ring closure in the intermediate $A$. The ease of cyclization of the latter is due to the spatial accessibility of the unsubstituted $\mathrm{N}$-nucleophilic center, and the experimentally found noticeably faster formation of thiazolidinones $\mathbf{1 1}$ as compared to thiazinanones $\mathbf{1 2}$ is explained by the proximity of the reaction centers upon creation of the 5-membered cycle. We were able to detect by ${ }^{31} \mathrm{P}$ NMR adduct $A$ (Scheme 6, $\mathrm{n}=2)$ resulting from addition of 3-mercaptopropionic acid $\left(\delta_{P} 16 \mathrm{ppm}\right)$. 


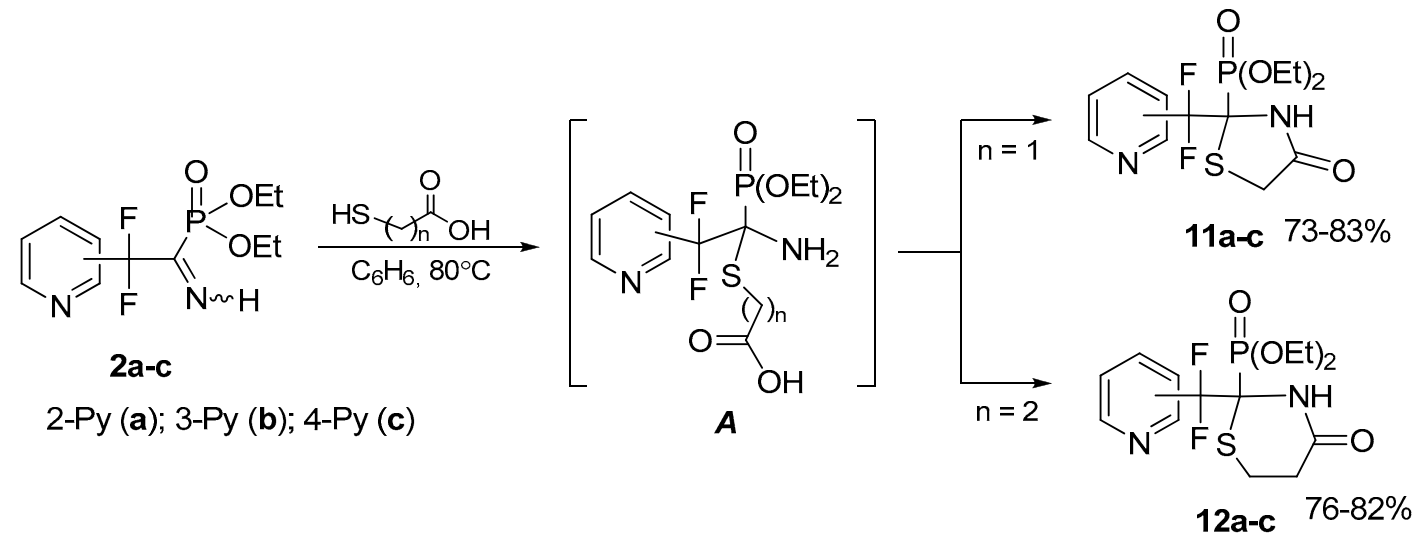

Scheme 6. Cyclocondensation with mercaptocarboxylic acids.

\section{Conclusions}

In summary, the triethylamine catalyzed addition of diethyl phosphite to isomeric pyridyldifluoroacetonitriles leads to $\alpha$-iminopyridyldifluoroethyl phosphonates, existing as equilibrium mixture of $E-Z$ isomers. The synthetic potential of these novel electrophilic building blocks was demonstrated by their reduction to biorelevant compounds combining in their structure aminophosphonic residue, difluoromethyl group and isomeric pyridine or piperidine moiety. Cyclocondensation with mercaptocarboxylic acids allow preparation of hybrid molecules bearing two bioactive heterocyclic moieties (pyridine and thiazolidine or thiazinane) and aminophosphonic fragment in a single molecular platform. These reactions proceed under mild neutral conditions and lead directly to $\mathrm{N}$-unprotected aminophosphonic derivatives.

\section{Materials and Methods}

${ }^{1} \mathrm{H},{ }^{19} \mathrm{~F}$, and ${ }^{13} \mathrm{C}$ NMR spectra were recorded using Bruker Avance NMR spectrometers operating at 302,400 and $499.8{ }^{1} \mathrm{H}$ frequencies; 75.8, 125.7 and $150.8 \mathrm{MHz}$ for ${ }^{13} \mathrm{C}$ experiments; $188,376.5$ and $470.3 \mathrm{MHz}$ for ${ }^{19} \mathrm{~F} ; 81$ and $202.3 \mathrm{MHz}$ for ${ }^{31} \mathrm{P}$. Chemical shifts are reported relative to internal TMS $\left({ }^{1} \mathrm{H}\right)$ or $\mathrm{CFCl}_{3}\left({ }^{19} \mathrm{~F}\right)$ and external $85 \%-\mathrm{H}_{3} \mathrm{PO}_{4}\left({ }^{31} \mathrm{P}\right)$ standards. Melting points are uncorrected. Solvents were dried before use according to standard methods. Elemental analysis was carried out in the analytical laboratory of Institute of Organic Chemistry, NAS of Ukraine. See the Supplementary File for the ${ }^{1} \mathrm{H},{ }^{19} \mathrm{~F}$, and ${ }^{13} \mathrm{C}$ NMR spectra of compounds synthesized.

\subsection{Synthesis of Diethyl (2,2-Difluoro-1-imino-2-(pyridinyl)ethyl)phosphonates 2a-c: General Procedure}

To equimolar mixture of pyridinylacetonitrile $1(0.45 \mathrm{~g}, 2.9 \mathrm{mmol})$ and diethyl phosphite $(0.4 \mathrm{~g}, 2.9 \mathrm{mmol})$ was added triethylamine $(0.58 \mathrm{mmol}, 0.059 \mathrm{~g})$. After $12 \mathrm{~h}$ for $\mathbf{2 b , c}$ and $120 \mathrm{~h}$ for $2 \mathrm{a}$ triethylamine was removed under vacuum.

\subsubsection{Diethyl (2,2-Difluoro-1-imino-2-(pyridin-2-yl)ethyl)phosphonate 2a}

Yield $0.85 \mathrm{~g}(100 \%)$; light brown oil. $\mathrm{Z} / \mathrm{E}=2: 1$. Z-isomer: ${ }^{1} \mathrm{H} \mathrm{NMR}\left(499.8 \mathrm{MHz}, \mathrm{CDCl}_{3}\right)$ $\delta$ (ppm): $1.33\left(\mathrm{t},{ }^{3} J_{\mathrm{HH}}=7.2 \mathrm{~Hz}, 6 \mathrm{H}, \mathrm{CH}_{3}\right), 4.16-4.22\left(\mathrm{~m}, 4 \mathrm{H}, \mathrm{CH}_{2}\right), 7.40\left(\mathrm{t},{ }^{3} J_{\mathrm{HH}}=7.9 \mathrm{~Hz}, 1 \mathrm{H}\right.$, Py), $7.74\left(\mathrm{~d},{ }^{3} J_{\mathrm{HH}}=7.9 \mathrm{~Hz}, 1 \mathrm{H}, \mathrm{Py}\right), 7.84-7.88(\mathrm{~m}, 1 \mathrm{H}, \mathrm{Py}), 8.66\left(\mathrm{~d},{ }^{3} J_{\mathrm{HH}}=4.9 \mathrm{~Hz}, 1 \mathrm{H}, \mathrm{Py}\right)$, $12.27\left(\mathrm{~d},{ }^{3} J_{\mathrm{HP}}=39.9 \mathrm{~Hz}, 1 \mathrm{H}, \mathrm{NH}\right)$.

${ }^{13} \mathrm{C}$ NMR $\left(75.8 \mathrm{MHz}, \mathrm{CDCl}_{3}\right) \delta(\mathrm{ppm}): 16.0\left(\mathrm{~d},{ }^{3} J_{\mathrm{CP}}=6 \mathrm{~Hz}, \mathrm{CH}_{3}\right), 63.8\left(\mathrm{~d},{ }^{2} J_{\mathrm{CP}}=6 \mathrm{~Hz}\right.$, $\left.\mathrm{CH}_{2}\right), 116.1\left(\mathrm{td},{ }^{1} J_{\mathrm{CF}}=246 \mathrm{~Hz},{ }^{2} J_{\mathrm{CP}}=33 \mathrm{~Hz}, \mathrm{CF}_{2}\right), 120.9\left(\mathrm{t},{ }^{3} J_{\mathrm{CF}}=4 \mathrm{~Hz}, \mathrm{C}_{\mathrm{Py}}\right), 125.1(\mathrm{~s}$, $\left.\mathrm{C}_{\mathrm{Py}}\right), 136.9\left(\mathrm{~s}, \mathrm{C}_{\mathrm{Py}}\right), 149.3\left(\mathrm{~s}, \mathrm{C}_{\mathrm{Py}}\right), 152.8\left(\mathrm{t},{ }^{2} J_{\mathrm{CF}}=28.5 \mathrm{~Hz}, \underline{C C F}_{2}\right), 173.2\left(\mathrm{dt},{ }^{1} J_{\mathrm{CP}}=153 \mathrm{~Hz}\right.$, $\left.{ }^{2} J_{\mathrm{CF}}=33 \mathrm{~Hz}, \mathrm{C}=\mathrm{N}\right)$.

${ }^{19} \mathrm{~F}$ NMR $\left(470.3 \mathrm{MHz}, \mathrm{CDCl}_{3}\right) \delta(\mathrm{ppm}):-100.2$.

${ }^{31} \mathrm{P}$ NMR $\left(202.3 \mathrm{MHz}, \mathrm{CDCl}_{3}\right) \delta$ (ppm): $5.9\left(\mathrm{~m},{ }^{3} J_{\mathrm{PH}}=39.9 \mathrm{~Hz}\right)$. 
E-isomer: ${ }^{1} \mathrm{H}$ NMR $\left(499.8 \mathrm{MHz}, \mathrm{CDCl}_{3}\right) \delta(\mathrm{ppm}): 1.26\left(\mathrm{t},{ }^{3} J_{\mathrm{HH}}=7.1 \mathrm{~Hz}, 6 \mathrm{H}, \mathrm{CH}_{3}\right)$, 4.10-4.15 (m, 4H, CH $), 7.40\left(\mathrm{t}^{3}{ }^{3} \mathrm{HH}_{\mathrm{HH}}=7.7 \mathrm{~Hz}, 1 \mathrm{H}, \mathrm{Py}\right), 7.80-7.84(\mathrm{~m}, 2 \mathrm{H}, \mathrm{Py}), 8.6(\mathrm{~d}$, $\left.{ }^{3} J_{\mathrm{HH}}=4.9 \mathrm{~Hz}, 1 \mathrm{H}, \mathrm{Py}\right), 11.97\left(\mathrm{dt},{ }^{3} J_{\mathrm{HP}}=61.6 \mathrm{~Hz},{ }^{4} J_{\mathrm{HF}}=4.0 \mathrm{~Hz}, 1 \mathrm{H}, \mathrm{NH}\right)$.

${ }^{13} \mathrm{C}$ NMR $\left(75.8 \mathrm{MHz}, \mathrm{CDCl}_{3}\right) \delta(\mathrm{ppm}): 16.2\left(\mathrm{~d},{ }^{3} J_{\mathrm{CP}}=6 \mathrm{~Hz}, \mathrm{CH}_{3}\right), 63.7\left(\mathrm{~d},{ }^{2} J_{\mathrm{CP}}=6 \mathrm{~Hz}\right.$, $\left.\mathrm{CH}_{2}\right), 113.9\left(\mathrm{td},{ }^{1} J_{\mathrm{CF}}=249 \mathrm{~Hz},{ }^{2} J_{\mathrm{CP}}=42 \mathrm{~Hz}, \mathrm{CF}_{2}\right), 120.8\left(\mathrm{t},{ }^{3} J_{\mathrm{CF}}=4 \mathrm{~Hz}, \mathrm{C}_{\mathrm{Py}}\right), 125.4(\mathrm{~s}$,

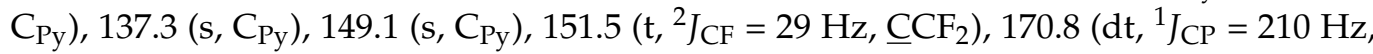
$\left.{ }^{2} J_{\mathrm{CF}}=30 \mathrm{~Hz}, \mathrm{C}=\mathrm{N}\right)$.

${ }^{19} \mathrm{~F}$ NMR $\left(470.3 \mathrm{MHz}, \mathrm{CDCl}_{3}\right) \delta(\mathrm{ppm}):-103.5$.

${ }^{31} \mathrm{P}$ NMR (202.3 MHz, $\left.\mathrm{CDCl}_{3}\right) \delta(\mathrm{ppm}): 6.6\left(\mathrm{~m},{ }^{3} J_{\mathrm{PH}}=61.6 \mathrm{~Hz}\right)$.

Anal. Calc. for $\mathrm{C}_{11} \mathrm{H}_{15} \mathrm{~F}_{2} \mathrm{~N}_{2} \mathrm{O}_{3} \mathrm{P}: \mathrm{C}, 45.21 ; \mathrm{H}, 5.17 ; \mathrm{N}, 9.59$. Found: $\mathrm{C}, 45.38 ; \mathrm{H}, 5.29$; $\mathrm{N}, 9.67$.

\subsubsection{Diethyl (2,2-Difluoro-1-imino-2-(pyridin-3-yl)ethyl)phosphonate $\mathbf{2 b}$}

Yield $0.85 \mathrm{~g}(100 \%)$; yellow oil. $\mathrm{Z} / E=6: 1$. $Z$-isomer: ${ }^{1} \mathrm{H}$ NMR $\left(302 \mathrm{MHz}, \mathrm{CDCl}_{3}\right) \delta$ (ppm): $1.27\left(\mathrm{t}^{3}{ }^{3} \mathrm{H}_{\mathrm{HH}}=7.1 \mathrm{~Hz}, 6 \mathrm{H}, \mathrm{CH}_{3}\right), 3.93-4.20\left(\mathrm{~m}, 4 \mathrm{H}, \mathrm{CH}_{2}\right), 7.29\left(\mathrm{dd}^{3}{ }^{3} J_{\mathrm{HH}}=8.1 \mathrm{~Hz}\right.$, $\left.{ }^{3} J_{\mathrm{HH}}=5.1 \mathrm{~Hz}, \mathrm{Py}\right), 7.81\left(\mathrm{~d},{ }^{3} J_{\mathrm{HH}}=8.1 \mathrm{~Hz}, 1 \mathrm{H}, \mathrm{Py}\right), 8.63\left(\mathrm{~d},{ }^{3} J_{\mathrm{HH}}=5.1 \mathrm{~Hz}, 1 \mathrm{H}, \mathrm{Py}\right), 8.74(\mathrm{~s}$, $1 \mathrm{H}, \mathrm{Py}), 12.17\left(\mathrm{~d},{ }^{3} J_{\mathrm{HP}}=39.6 \mathrm{~Hz}, 1 \mathrm{H}, \mathrm{NH}\right)$.

${ }^{13} \mathrm{C}$ NMR $\left(75.8 \mathrm{MHz}, \mathrm{CDCl}_{3}\right) \delta(\mathrm{ppm}): 16.1\left(\mathrm{~d},{ }^{3} J_{\mathrm{CP}}=6 \mathrm{~Hz}, \mathrm{CH}_{3}\right), 63.9\left(\mathrm{~d},{ }^{2} J_{\mathrm{CP}}=6 \mathrm{~Hz}\right.$, $\left.\mathrm{CH}_{2}\right), 117.3\left(\mathrm{td},{ }^{1} J_{\mathrm{CF}}=246 \mathrm{~Hz},{ }^{2} J_{\mathrm{CP}}=35 \mathrm{~Hz}, \mathrm{CF}_{2}\right), 122.9\left(\mathrm{~s}, \mathrm{C}_{\mathrm{Py}}\right), 129.9\left(\mathrm{t},{ }^{2} J_{\mathrm{CF}}=27 \mathrm{~Hz}\right.$, $\left.\underline{C} F_{2}\right), 133.8\left(t,{ }^{3} J_{\mathrm{CF}}=6 \mathrm{~Hz}, \mathrm{C}_{\mathrm{Py}}\right), 147.3\left(\mathrm{t},{ }^{3} J_{\mathrm{CF}}=6.5 \mathrm{~Hz}, \mathrm{C}_{\mathrm{Py}}\right), 151.6\left(\mathrm{~s}, \mathrm{C}_{\mathrm{Py}}\right), 173.0(\mathrm{dt}$, $\left.{ }^{1} J_{\mathrm{CP}}=154 \mathrm{~Hz},{ }^{2} J_{\mathrm{CF}}=35 \mathrm{~Hz}, \mathrm{C}=\mathrm{N}\right)$.

${ }^{19} \mathrm{~F}$ NMR $\left(470.3 \mathrm{MHz}, \mathrm{CDCl}_{3}\right) \delta(\mathrm{ppm}):-97.5$

${ }^{31} \mathrm{P}$ NMR $\left(81 \mathrm{MHz}, \mathrm{CDCl}_{3}\right) \delta(\mathrm{ppm}): 1.2\left(\mathrm{~m},{ }^{3} J_{\mathrm{PH}}=39.6 \mathrm{~Hz}\right)$.

E-isomer: ${ }^{1} \mathrm{H}$ NMR $\left(302 \mathrm{MHz}, \mathrm{CDCl}_{3}\right) \delta(\mathrm{ppm}): 1.15\left(\mathrm{t},{ }^{3} \mathrm{JHH}_{\mathrm{HH}}=6.9 \mathrm{~Hz}, 6 \mathrm{H}, \mathrm{CH}_{3}\right)$, 3.93-4.20 (m, 4H, $\left.\mathrm{CH}_{2}\right), 7.30-7.35(\mathrm{~m}, 1 \mathrm{H}, \mathrm{Py}), 7.85\left(\mathrm{~d},{ }^{3} J_{\mathrm{HH}}=6.7 \mathrm{~Hz}, 1 \mathrm{H}, \mathrm{Py}\right), 8.66(\mathrm{~d}$, $\left.{ }^{3} J_{\mathrm{HH}}=7.2 \mathrm{~Hz}, 1 \mathrm{H}, \mathrm{Py}\right), 8.78(\mathrm{~s}, 1 \mathrm{H}, \mathrm{Py}), 11.85\left(\mathrm{~d},{ }^{3} J_{\mathrm{HP}}=61.2 \mathrm{~Hz}, 1 \mathrm{H}, \mathrm{NH}\right)$.

${ }^{19} \mathrm{~F}$ NMR $\left(470.3 \mathrm{MHz}, \mathrm{CDCl}_{3}\right) \delta(\mathrm{ppm}):-100.5$.

${ }^{31} \mathrm{P} \mathrm{NMR}\left(81 \mathrm{MHz}, \mathrm{CDCl}_{3}\right) \delta$ (ppm): $2.9\left(\mathrm{~m},{ }^{3} J_{\mathrm{PH}}=61.2 \mathrm{~Hz}\right)$.

Anal. Calc. for $\mathrm{C}_{11} \mathrm{H}_{15} \mathrm{~F}_{2} \mathrm{~N}_{2} \mathrm{O}_{3} \mathrm{P}: \mathrm{C}, 45.21 ; \mathrm{H}, 5.17 ; \mathrm{N}, 9.59$. Found: $\mathrm{C}, 45.46 ; \mathrm{H}, 5.04$; $\mathrm{N}, 9.43$.

\subsubsection{Diethyl (2,2-Difluoro-1-imino-2-(pyridin-4-yl)ethyl)phosphonate 2c}

Yield $0.85 \mathrm{~g}(100 \%)$; light orange oil. $\mathrm{Z} / \mathrm{E}=7: 1 . \mathrm{Z}$-isomer: ${ }^{1} \mathrm{H} \mathrm{NMR}\left(400 \mathrm{MHz}, \mathrm{CDCl}_{3}\right)$ $\delta$ (ppm): $1.34\left(\mathrm{t},{ }^{3} J_{\mathrm{HH}}=7.1 \mathrm{~Hz}, 6 \mathrm{H}, \mathrm{CH}_{3}\right), 4.14-4.27\left(\mathrm{~m}, 4 \mathrm{H}, \mathrm{CH}_{2}\right), 7.50\left(\mathrm{~d},{ }^{3} J_{\mathrm{HH}}=6 \mathrm{~Hz}, 2 \mathrm{H}\right.$, Py), $8.74\left(\mathrm{~d},{ }^{3} J_{\mathrm{HH}}=6 \mathrm{~Hz}, 2 \mathrm{H}, \mathrm{Py}\right), 12.21\left(\mathrm{~d},{ }^{3} J_{\mathrm{HP}}=39.5 \mathrm{~Hz}, 1 \mathrm{H}, \mathrm{NH}\right)$.

${ }^{13} \mathrm{C}$ NMR $\left(75.8 \mathrm{MHz}, \mathrm{CDCl}_{3}\right) \delta(\mathrm{ppm}): 16.3\left(\mathrm{~d},{ }^{3} J_{\mathrm{CP}}=6 \mathrm{~Hz}, \mathrm{CH}_{3}\right), 64.1\left(\mathrm{~d},{ }^{2} J_{\mathrm{CP}}=6 \mathrm{~Hz}\right.$, $\left.\mathrm{CH}_{2}\right), 116.9\left(\mathrm{td},{ }^{1} J_{\mathrm{CF}}=246 \mathrm{~Hz},{ }^{2} J_{\mathrm{CP}}=34 \mathrm{~Hz}, \mathrm{CF}_{2}\right), 120.4\left(\mathrm{t},{ }^{3} J_{\mathrm{CF}}=6 \mathrm{~Hz}, 2 \mathrm{C}_{\mathrm{Py}}\right), 142.2(\mathrm{td}$, $\left.{ }^{2} J_{\mathrm{CF}}=27 \mathrm{~Hz},{ }^{3} J_{\mathrm{CP}}=6 \mathrm{~Hz}, \mathrm{CCF}_{2}\right), 150.2\left(\mathrm{~s}, 2 \mathrm{C}_{\mathrm{Py}}\right), 172.9\left(\mathrm{dt},{ }^{1} J_{\mathrm{CP}}=154 \mathrm{~Hz},{ }^{2} J_{\mathrm{CF}}=35 \mathrm{~Hz}\right.$, $\mathrm{C}=\mathrm{N})$.

${ }^{19} \mathrm{~F}$ NMR $\left(470.3 \mathrm{MHz}, \mathrm{CDCl}_{3}\right) \delta(\mathrm{ppm}):-99.4$.

${ }^{31} \mathrm{P} \mathrm{NMR}\left(202.3 \mathrm{MHz}, \mathrm{CDCl}_{3}\right) \delta(\mathrm{ppm}): 0.1\left(\mathrm{~m},{ }^{3} J_{\mathrm{PH}}=39.5 \mathrm{~Hz}\right)$.

E-isomer: ${ }^{1} \mathrm{H}$ NMR $\left(400 \mathrm{MHz}, \mathrm{CDCl}_{3}\right) \delta(\mathrm{ppm}): 1.26\left(\mathrm{t},{ }^{3} \mathrm{~J}_{\mathrm{HH}}=7.1 \mathrm{~Hz}, 6 \mathrm{H}, \mathrm{CH}_{3}\right)$, 4.05-4.13 (m, 4H, CH $), 7.45\left(\mathrm{~d},{ }^{3} \mathrm{~J}_{\mathrm{HH}}=5 \mathrm{~Hz}, 2 \mathrm{H}, \mathrm{Py}\right), 8.76\left(\mathrm{~d},{ }^{3} J_{\mathrm{HH}}=5 \mathrm{~Hz}, 2 \mathrm{H}, \mathrm{Py}\right), 11.81$ $\left(\mathrm{d},{ }^{3} J_{\mathrm{HP}}=60.9 \mathrm{~Hz}, 1 \mathrm{H}, \mathrm{NH}\right)$.

${ }^{19} \mathrm{~F}$ NMR $\left(470.3 \mathrm{MHz}, \mathrm{CDCl}_{3}\right) \delta(\mathrm{ppm}):-102.4$.

${ }^{31} \mathrm{P}$ NMR $\left(202.3 \mathrm{MHz}, \mathrm{CDCl}_{3}\right) \delta(\mathrm{ppm}): 1.8\left(\mathrm{~m},{ }^{3} J_{\mathrm{PH}}=60.9 \mathrm{~Hz}\right)$.

Anal. Calc. for $\mathrm{C}_{11} \mathrm{H}_{15} \mathrm{~F}_{2} \mathrm{~N}_{2} \mathrm{O}_{3} \mathrm{P}: \mathrm{C}, 45.21 ; \mathrm{H}, 5.17 ; \mathrm{N}, 9.59$. Found: $\mathrm{C}, 45.03 ; \mathrm{H}, 5.23$; N, 9.41.

\subsection{Reactions of Iminophosphonates $\mathbf{2} \mathbf{a}-\mathbf{c}$ with Methanol}

Iminophosphonates $\mathbf{2 a - c}(0.02 \mathrm{~g}, 0.07 \mathrm{mmol})$ were dissolved in methanol $(0.6 \mathrm{~mL})$ at room temperature. According to ${ }^{31} \mathrm{P},{ }^{19} \mathrm{~F}$ NMR the reaction was completed after $8 \mathrm{~h}(\mathbf{2 a})$ or 2 days $(\mathbf{2} \mathbf{b}, \mathbf{c})$. The spectral data are similar to those of methanol adduct with trifluoromethyl analog [17]. 
Spectral data for adduct 6a: ${ }^{1} \mathrm{H}$ NMR $\left(400 \mathrm{MHz}, \mathrm{CDCl}_{3}\right) \delta(\mathrm{ppm}): 1.30-1.34(\mathrm{~m}, 6 \mathrm{H}$, $\left.\mathrm{CH}_{3}\right), 3.21\left(\mathrm{~s}, 3 \mathrm{H}, \mathrm{OCH}_{3}\right), 4.20-4.30\left(\mathrm{~m}, 4 \mathrm{H}, \mathrm{CH}_{2}\right), 7.36\left(\mathrm{dd},{ }^{3} J_{\mathrm{HH}}=8.1 \mathrm{~Hz},{ }^{3} J_{\mathrm{HH}}=3.9 \mathrm{~Hz}, 1 \mathrm{H}\right.$, Py), $7.68\left(\mathrm{~d},{ }^{3} J_{\mathrm{HH}}=8.1 \mathrm{~Hz}, 1 \mathrm{H}, \mathrm{Py}\right), 7.78\left(\mathrm{t},{ }^{3} J_{\mathrm{HH}}=8.1 \mathrm{~Hz}, 1 \mathrm{H}, \mathrm{Py}\right), 8.60\left(\mathrm{~d},{ }^{3} J_{\mathrm{HH}}=3.9 \mathrm{~Hz}\right.$, $1 \mathrm{H}, \mathrm{Py}) .{ }^{19} \mathrm{~F}$ NMR $(470.3 \mathrm{MHz}, \mathrm{MeOH}) \delta(\mathrm{ppm}):-103.0\left(\mathrm{~d},{ }^{2} J_{\mathrm{FAFB}}=255 \mathrm{~Hz}\right),-110.5(\mathrm{~d}$, $\left.{ }^{2} J_{\mathrm{FBFA}}=255 \mathrm{~Hz}\right) \cdot{ }^{31} \mathrm{PNMR}(202.3 \mathrm{MHz}, \mathrm{MeOH}) \delta(\mathrm{ppm}): 15.1$.

Spectral data for adduct $6 \mathbf{b}:{ }^{1} \mathrm{H} \mathrm{NMR}\left(400 \mathrm{MHz}, \mathrm{CDCl}_{3}\right) \delta(\mathrm{ppm}): 1.09\left(\mathrm{t}^{3}{ }^{3} \mathrm{HH}=7.5 \mathrm{~Hz}\right.$, $\left.3 \mathrm{H}, \mathrm{CH}_{3}\right), 1.27\left(\mathrm{t},{ }^{3} J_{\mathrm{HH}}=7.6 \mathrm{~Hz}, 3 \mathrm{H}, \mathrm{CH}_{3}\right), 2.40\left(\mathrm{~d},{ }^{3} \mathrm{JP}_{\mathrm{HP}}=20 \mathrm{~Hz}, 2 \mathrm{H}, \mathrm{NH}_{2}\right), 3.37(\mathrm{~s}, 3 \mathrm{H}$, $\left.\mathrm{OCH}_{3}\right), 3.94-4.08\left(\mathrm{~m}, 2 \mathrm{H}, \mathrm{OCH}_{2}\right), 4.13-4.18\left(\mathrm{~m}, 2 \mathrm{H}, \mathrm{OCH}_{2}\right), 7.30\left(\mathrm{dd},{ }^{3} \mathrm{~J}_{\mathrm{HH}}=7.3 \mathrm{~Hz}\right.$, $\left.{ }^{3} J_{\mathrm{HH}}=4.2 \mathrm{~Hz}, 1 \mathrm{H}, \mathrm{Py}\right), 7.87\left(\mathrm{~d},{ }^{3} J_{\mathrm{HH}}=7.3 \mathrm{~Hz}, 1 \mathrm{H}, \mathrm{Py}\right), 8.63\left(\mathrm{~d},{ }^{3} J_{\mathrm{HH}}=4.2 \mathrm{~Hz}, 1 \mathrm{H}, \mathrm{Py}\right), 8.77$ (s, $1 \mathrm{H}, \mathrm{Py}) .{ }^{19} \mathrm{~F}$ NMR $(470.3 \mathrm{MHz}, \mathrm{MeOH}) \delta(\mathrm{ppm}):-103.9\left(\mathrm{~d},{ }^{2} J_{\mathrm{FAFB}}=257 \mathrm{~Hz}\right),-106.6(\mathrm{dd}$, $\left.{ }^{2} J_{\mathrm{FBFA}}=257 \mathrm{~Hz},{ }^{3} J_{\mathrm{FP}}=7 \mathrm{~Hz}\right) .{ }^{31} \mathrm{PNMR}(202.3 \mathrm{MHz}, \mathrm{MeOH}) \delta(\mathrm{ppm}): 14.8\left(\mathrm{~m},{ }^{3} J_{\mathrm{PF}}=7 \mathrm{~Hz}\right)$

Spectral data for adduct $6 \mathrm{c}:{ }^{1} \mathrm{H}$ NMR $\left(400 \mathrm{MHz}, \mathrm{CDCl}_{3}\right) \delta(\mathrm{ppm}): 1.13\left(\mathrm{t}^{3}{ }^{3} \mathrm{~J}_{\mathrm{HH}}=6.9 \mathrm{~Hz}\right.$, $\left.3 \mathrm{H}, \mathrm{CH}_{3}\right), 1.30\left(\mathrm{t},{ }^{3} J_{\mathrm{HH}}=6.6 \mathrm{~Hz}, 3 \mathrm{H}, \mathrm{CH}_{3}\right), 2.40\left(\mathrm{~d},{ }^{3} J_{\mathrm{HP}}=22.5 \mathrm{~Hz}, 2 \mathrm{H}, \mathrm{NH}_{2}\right), 3.38(\mathrm{~s}, 3 \mathrm{H}$, $\left.\mathrm{OCH}_{3}\right), 3.97-4.12\left(\mathrm{~m}, 2 \mathrm{H}, \mathrm{OCH}_{2}\right), 4.13-4.21\left(\mathrm{~m}, 2 \mathrm{H}, \mathrm{OCH}_{2}\right), 7.49\left(\mathrm{~d},{ }^{3} \mathrm{~J}_{\mathrm{HH}}=5.3 \mathrm{~Hz}, 2 \mathrm{H}\right.$, Py), $8.66\left(\mathrm{~d},{ }^{3} J_{\mathrm{HH}}=5.3 \mathrm{~Hz}, 2 \mathrm{H}, \mathrm{Py}\right) .{ }^{19} \mathrm{~F}$ NMR $(470.3 \mathrm{MHz}, \mathrm{MeOH}) \delta(\mathrm{ppm}):-106.4(\mathrm{~d}$, $\left.{ }^{2} J_{\mathrm{FAFB}}=254 \mathrm{~Hz}\right),-108.4\left(\mathrm{dd},{ }^{2} J_{\mathrm{FBFA}}=254 \mathrm{~Hz},{ }^{3} J_{\mathrm{FP}}=7 \mathrm{~Hz}\right) .{ }^{31} \mathrm{PNMR}(202.3 \mathrm{MHz}, \mathrm{MeOH}) \delta$ (ppm): $13.1\left(\mathrm{~m},{ }^{3} J_{\mathrm{PF}}=7 \mathrm{~Hz}\right)$.

\subsection{Synthesis of Diethyl (1-Amino-2,2-difluoro-2-(pyridinyl)ethyl)phosphonates 7a-c: General Procedure}

To a solution of respective iminophosphonate $2(0.65 \mathrm{~g}, 2.22 \mathrm{mmol})$ in THF $(8 \mathrm{~mL})$ borane-dimethyl sulfide complex $(0.25 \mathrm{~g}$, $3.34 \mathrm{mmol})$ was added dropwise at $-30{ }^{\circ} \mathrm{C}$ in argon atmosphere. The reaction mixture was kept at this temperature for $0.5 \mathrm{~h}$ and allowed to warm to r.t. After that the reaction mixture was quenched with methanol $(2 \mathrm{~mL})$ and stirred for $15 \mathrm{~min}$. Then solvents were evaporated under reduced pressure and residue was dried in vacuo.

\subsubsection{Diethyl (1-Amino-2,2-difluoro-2-(pyridin-2-yl)ethyl)phosphonate 7a}

Yield $0.65 \mathrm{~g}(100 \%)$; yellow crystals; $\mathrm{mp} 52-53{ }^{\circ} \mathrm{C} .{ }^{1} \mathrm{H}$ NMR $\left(499.8 \mathrm{MHz}, \mathrm{CDCl}_{3}\right) \delta$ (ppm): $1.22\left(\mathrm{t},{ }^{3} \mathrm{~J}_{\mathrm{HH}}=7.1 \mathrm{~Hz}, 3 \mathrm{H}, \mathrm{CH}_{3}\right), 1.25\left(\mathrm{t},{ }^{3} \mathrm{~J}_{\mathrm{HH}}=7.1 \mathrm{~Hz}, 3 \mathrm{H}, \mathrm{CH}_{3}\right), 1.85(\mathrm{br} \mathrm{s}, 2 \mathrm{H}$, $\left.\mathrm{NH}_{2}\right), 4.18-4.00\left(\mathrm{~m}, 4 \mathrm{H}, \mathrm{CH}_{2}\right), 4.23(\mathrm{~m}, 1 \mathrm{H}, \mathrm{CH}), 7.37\left(\mathrm{dd},{ }^{3} J_{\mathrm{HH}}=7.8,{ }^{3} J_{\mathrm{HH}}=4.8 \mathrm{~Hz}, 1 \mathrm{H}, \mathrm{Py}\right)$, $7.7\left(\mathrm{~d},{ }^{3} J_{\mathrm{HH}}=7.8 \mathrm{~Hz}, 1 \mathrm{H}, \mathrm{Py}\right), 7.81\left(\mathrm{t},{ }^{3} J_{\mathrm{HH}}=7.8 \mathrm{~Hz}, 1 \mathrm{H}, \mathrm{Py}\right), 8.64\left(\mathrm{~d},{ }^{3} J_{\mathrm{HH}}=4.8 \mathrm{~Hz}, 1 \mathrm{H}, \mathrm{Py}\right)$ ${ }^{13} \mathrm{C}$ NMR $\left(125.7 \mathrm{MHz}, \mathrm{CDCl}_{3}\right) \delta(\mathrm{ppm}): 16.3\left(\mathrm{~d},{ }^{3} J_{\mathrm{CP}}=5.9 \mathrm{~Hz}, \mathrm{CH}_{3}\right), 16.4\left(\mathrm{~d},{ }^{3} J_{\mathrm{CP}}=6.1 \mathrm{~Hz}\right.$, $\left.\mathrm{CH}_{3}\right), 53.7\left(\mathrm{dt},{ }^{1} J_{\mathrm{CP}}=154.9 \mathrm{~Hz},{ }^{2} J_{\mathrm{CF}}=28 \mathrm{~Hz}, \mathrm{CH}\right), 62.7\left(\mathrm{~d},{ }^{2} J_{\mathrm{CP}}=6.9 \mathrm{~Hz}, \mathrm{CH}_{2}\right), 63.0(\mathrm{~d}$, $\left.{ }^{2} J_{\mathrm{CP}}=6.7 \mathrm{~Hz}, \mathrm{CH}_{2}\right), 120.2\left(\mathrm{td},{ }^{1} J_{\mathrm{CF}}=248 \mathrm{~Hz},{ }^{2} J_{\mathrm{CP}}=5 \mathrm{~Hz}, \mathrm{CF}_{2}\right), 121.0\left(\mathrm{t},{ }^{3} J_{\mathrm{CF}}=4.8 \mathrm{~Hz}\right.$, $\left.\mathrm{C}_{\mathrm{Py}}\right), 124.9\left(\mathrm{~s}, \mathrm{C}_{\mathrm{Py}}\right), 137.0\left(\mathrm{~s}, \mathrm{C}_{\mathrm{Py}}\right), 149.2\left(\mathrm{~s}, \mathrm{C}_{\mathrm{Py}}\right), 153.6\left(\mathrm{t},{ }^{2} J_{\mathrm{CF}}=29 \mathrm{~Hz}, \underline{C C F}_{2}\right) .{ }^{19} \mathrm{~F} \mathrm{NMR}$ $\left(470.3 \mathrm{MHz}, \mathrm{CDCl}_{3}\right) \delta(\mathrm{ppm}):-103.8\left(\mathrm{ddd},{ }^{2} J_{\mathrm{FAFB}}=256.5 \mathrm{~Hz},{ }^{3} J_{\mathrm{FH}}=15.4 \mathrm{~Hz},{ }^{3} J_{\mathrm{FP}}=15.4 \mathrm{~Hz}\right)$, $-106.3\left(\mathrm{dd},{ }^{2} J_{\mathrm{FBFA}}=256.5 \mathrm{~Hz},{ }^{3} \mathrm{~J}=15.1 \mathrm{~Hz}\right) .{ }^{31} \mathrm{P} \mathrm{NMR}\left(202.3 \mathrm{MHz}, \mathrm{CDCl}_{3}\right) \delta(\mathrm{ppm}): 19.8$. Anal. Calc. for $\mathrm{C}_{11} \mathrm{H}_{17} \mathrm{~F}_{2} \mathrm{~N}_{2} \mathrm{O}_{3} \mathrm{P}: \mathrm{C}, 44.90 ; \mathrm{H}, 5.82 ; \mathrm{N}$, 9.52. Found: $\mathrm{C}, 44.67 ; \mathrm{H}, 5.73 ; \mathrm{N}, 9.41$.

\subsubsection{Diethyl (1-Amino-2,2-difluoro-2-(pyridin-3-yl)ethyl)phosphonate $7 \mathbf{b}$}

Yield $0.65 \mathrm{~g}(100 \%)$; white-yellow oil. ${ }^{1} \mathrm{H}$ NMR $\left(302 \mathrm{MHz}, \mathrm{CDCl}_{3}\right) \delta$ (ppm): 1.13-1.43 $\left(\mathrm{m}, 6 \mathrm{H}, \mathrm{CH}_{3}\right), 1.85\left(\mathrm{br} \mathrm{s}, 2 \mathrm{H}, \mathrm{NH}_{2}\right), 3.62(\mathrm{~m}, 1 \mathrm{H}, \mathrm{CH}), 4.00-4.25\left(\mathrm{~m}, 4 \mathrm{H}, \mathrm{CH}_{2}\right), 7.54-7.64(\mathrm{~m}$, $1 \mathrm{H}, \mathrm{Py}), 8.15\left(\mathrm{~d},{ }^{3} \mathrm{~J}_{\mathrm{HH}}=8.1 \mathrm{~Hz}, 1 \mathrm{H}, \mathrm{Py}\right), 8.68\left(\mathrm{~d},{ }^{3} J_{\mathrm{HH}}=5.8 \mathrm{~Hz}, 1 \mathrm{H}, \mathrm{Py}\right), 8.82(\mathrm{~s}, 1 \mathrm{H}, \mathrm{Py}) .{ }^{13} \mathrm{C}$ NMR (125.7 MHz, DMSO-d $\left.\mathrm{d}_{6}\right) \delta(\mathrm{ppm}): 16.0\left(\mathrm{~d},{ }^{3} J_{\mathrm{CP}}=5 \mathrm{~Hz}, \mathrm{CH}_{3}\right), 16.2\left(\mathrm{~d},{ }^{3} J_{\mathrm{CP}}=5 \mathrm{~Hz}, \mathrm{CH}_{3}\right)$, $53.8\left(\mathrm{dt},{ }^{1} J_{\mathrm{CP}}=150 \mathrm{~Hz},{ }^{2} J_{\mathrm{CF}}=29 \mathrm{~Hz}, \mathrm{CH}\right), 62.0\left(\mathrm{~d},{ }^{2} J_{\mathrm{CP}}=6 \mathrm{~Hz}, \mathrm{CH}_{2}\right), 62.5\left(\mathrm{~d},{ }^{2} J_{\mathrm{CP}}=6 \mathrm{~Hz}\right.$, $\left.\mathrm{CH}_{2}\right), 119.8\left(\mathrm{td},{ }^{1} J_{\mathrm{CF}}=248 \mathrm{~Hz},{ }^{2} J_{\mathrm{CP}}=6 \mathrm{~Hz}, \mathrm{CF}_{2}\right), 125.8\left(\mathrm{~s}, \mathrm{C}_{\mathrm{Py}}\right), 133.6\left(\mathrm{td},{ }^{2} J_{\mathrm{CF}}=28 \mathrm{~Hz}\right.$, $\left.{ }^{3} J_{\mathrm{CP}}=4 \mathrm{~Hz}, \underline{\mathrm{CCF}}_{2}\right), 138.0\left(\mathrm{t},{ }^{3} J_{\mathrm{CF}}=6 \mathrm{~Hz}, \mathrm{C}_{\mathrm{Py}}\right), 144.8\left(\mathrm{t},{ }^{3} J_{\mathrm{CF}}=6 \mathrm{~Hz}, \mathrm{C}_{\mathrm{Py}}\right), 148.4\left(\mathrm{~s}, \mathrm{C}_{\mathrm{Py}}\right)$. ${ }^{19} \mathrm{~F} \mathrm{NMR}\left(470.3 \mathrm{MHz}, \mathrm{CDCl}_{3}\right) \delta(\mathrm{ppm}):-100.0\left(\mathrm{ddd},{ }^{2} J_{\mathrm{FAFB}}=259.1 \mathrm{~Hz},{ }^{3} J_{\mathrm{FH}}=11.8 \mathrm{~Hz}\right.$, $\left.{ }^{3} J_{\mathrm{FP}}=11.8 \mathrm{~Hz}\right),-100.8\left(\mathrm{ddd},{ }^{2} J_{\mathrm{FBFA}}=259.1 \mathrm{~Hz},{ }^{3} J_{\mathrm{FH}}=12.4 \mathrm{~Hz},{ }^{3} J_{\mathrm{FP}}=12.4 \mathrm{~Hz}\right) .{ }^{31} \mathrm{P} \mathrm{NMR}$ (202.3 MHz, $\left.\mathrm{CDCl}_{3}\right) \delta(\mathrm{ppm}): 18.8$. Anal. Calc. for $\mathrm{C}_{11} \mathrm{H}_{17} \mathrm{~F}_{2} \mathrm{~N}_{2} \mathrm{O}_{3} \mathrm{P}: \mathrm{C}, 44.90 ; \mathrm{H}, 5.82 ; \mathrm{N}$, 9.52. Found: C, 44.71; H, 5.74; N, 9.39. 
4.3.3. Diethyl (1-Amino-2,2-difluoro-2-(pyridin-4-yl)ethyl)phosphonate 7c

Yield $0.65 \mathrm{~g}(100 \%)$; white-yellow powder; $\mathrm{mp} 105-107^{\circ} \mathrm{C} .{ }^{1} \mathrm{H} \mathrm{NMR}\left(302 \mathrm{MHz}, \mathrm{CDCl}_{3}\right)$ $\delta$ (ppm): 1.12-1.48 (m, 6H, CH $\mathrm{CH}_{3}, 1.81$ (br s, $\left.2 \mathrm{H}, \mathrm{NH}_{2}\right), 3.60(\mathrm{~m}, 1 \mathrm{H}, \mathrm{CH}), 4.00-4.23(\mathrm{~m}, 4 \mathrm{H}$, $\left.\mathrm{CH}_{2}\right), 7.70\left(\mathrm{~d},{ }^{3} \mathrm{~J}_{\mathrm{HH}}=6 \mathrm{~Hz}, 2 \mathrm{H}, \mathrm{Py}\right), 8.70\left(\mathrm{~d},{ }^{3} \mathrm{~J}_{\mathrm{HH}}=6 \mathrm{~Hz}, 2 \mathrm{H}, \mathrm{Py}\right) .{ }^{13} \mathrm{C} \mathrm{NMR}(150.8 \mathrm{MHz}$, $\left.\mathrm{CDCl}_{3}\right) \delta(\mathrm{ppm}): 16.3\left(\mathrm{~d},{ }^{3} J_{\mathrm{CP}}=5.9 \mathrm{~Hz}, \mathrm{CH}_{3}\right), 16.4\left(\mathrm{~d},{ }^{3} J_{\mathrm{CP}}=5.9 \mathrm{~Hz}, \mathrm{CH}_{3}\right), 53.4(\mathrm{dt}$, $\left.{ }^{1} J_{\mathrm{CP}}=151.3 \mathrm{~Hz},{ }^{2} J_{\mathrm{CF}}=29.2 \mathrm{~Hz}, \mathrm{CH}\right), 63.2\left(\mathrm{~d},{ }^{2} J_{\mathrm{CP}}=6.8 \mathrm{~Hz}, \mathrm{CH}_{2}\right), 63.5\left(\mathrm{~d},{ }^{2} J_{\mathrm{CP}}=6.8 \mathrm{~Hz}\right.$, $\left.\mathrm{CH}_{2}\right), 119.0\left(\mathrm{td},{ }^{1} J_{\mathrm{CF}}=248 \mathrm{~Hz},{ }^{2} J_{\mathrm{CP}}=5.7 \mathrm{~Hz}, \mathrm{CF}_{2}\right), 123.0\left(\mathrm{t},{ }^{3} J_{\mathrm{CF}}=5.9 \mathrm{~Hz}, \mathrm{C}_{\mathrm{Py}}\right), 146.5$ $\left(\mathrm{t},{ }^{2} J_{\mathrm{CF}}=28.2 \mathrm{~Hz}, \mathrm{CCF}_{2}\right), 147.5\left(\mathrm{~s}, \mathrm{C}_{\mathrm{Py}}\right) .{ }^{19} \mathrm{~F} \mathrm{NMR}\left(470.3 \mathrm{MHz}, \mathrm{CDCl}_{3}\right) \delta(\mathrm{ppm}):-102.4$ $\left(\mathrm{ddd},{ }^{2} J_{\mathrm{FAFB}}=249.4 \mathrm{~Hz},{ }^{3} J_{\mathrm{FH}}=11.4 \mathrm{~Hz},{ }^{3} J_{\mathrm{FP}}=11.4 \mathrm{~Hz}\right),-102.7\left(\mathrm{ddd},{ }^{2} J_{\mathrm{FBFA}}=249.4 \mathrm{~Hz}\right.$, $\left.{ }^{3} J_{\mathrm{FH}}=11.8 \mathrm{~Hz},{ }^{3} J_{\mathrm{FP}}=11.8 \mathrm{~Hz}\right) .{ }^{31} \mathrm{P} \mathrm{NMR}\left(202.3 \mathrm{MHz}, \mathrm{CDCl}_{3}\right) \delta(\mathrm{ppm}): 17.95\left(\mathrm{~m},{ }^{2} J_{\mathrm{PH}}=18.2\right.$, ${ }^{3} J_{\mathrm{PF}}=8.6 \mathrm{~Hz}$ ). Anal. Calc. for $\mathrm{C}_{11} \mathrm{H}_{17} \mathrm{~F}_{2} \mathrm{~N}_{2} \mathrm{O}_{3} \mathrm{P}: \mathrm{C}, 44.90 ; \mathrm{H}, 5.82 ; \mathrm{N}, 9.52$. Found: C, 44.78; $\mathrm{H}, 5.68 ; \mathrm{N}, 9.43$.

\subsection{Synthesis of (1-Amino-2,2-difluoro-2-(pyridinyl)ethyl)phosphonic Acids 8a-c: General Procedure}

To respective aminophosphonate $7(0.3 \mathrm{~g}, 1.02 \mathrm{mmol})$ was added concentrated $\mathrm{HCl}$ $(2 \mathrm{~mL})$, the reaction mixture was heated at $110{ }^{\circ} \mathrm{C}$ for $2.5 \mathrm{~h}$. After that $\mathrm{HCl} / \mathrm{H}_{2} \mathrm{O}$ was evaporated, the residue was dissolved in $\mathrm{MeOH}(2 \mathrm{~mL})$, and treated with propylene oxide $(0.35 \mathrm{~g}, 6.12 \mathrm{mmol})$. After $24 \mathrm{~h}$ the precipitate formed was separated by filtration, washed with $\mathrm{MeOH}(2 \mathrm{~mL})$ and dried in vacuo.

\subsection{1. (1-Amino-2,2-difluoro-2-(pyridin-2-yl)ethyl)phosphonic Acid 8a}

Yield $0.17 \mathrm{~g}(70 \%)$; white powder; $\mathrm{mp} 235-237^{\circ} \mathrm{C}$ (decomp.). ${ }^{1} \mathrm{H}$ NMR $(499.8 \mathrm{MHz}$, $\left.\mathrm{D}_{2} \mathrm{O}\right) \delta(\mathrm{ppm}): 4.13-4.30(\mathrm{~m}, 1 \mathrm{H}, \mathrm{CH}), 7.58-7.64(\mathrm{~m}, 1 \mathrm{H}, \mathrm{Py}), 7.86\left(\mathrm{~d},{ }^{3} \mathrm{~J}_{\mathrm{HH}}=8.2 \mathrm{~Hz}, 1 \mathrm{H}, \mathrm{Py}\right)$, $8.04\left(\mathrm{t},{ }^{3} \mathrm{~J}_{\mathrm{HH}}=8.2 \mathrm{~Hz}, 1 \mathrm{H}, \mathrm{Py}\right), 8.64\left(\mathrm{~d},{ }^{3} \mathrm{~J}_{\mathrm{HH}}=4.9 \mathrm{~Hz}, 1 \mathrm{H}, \mathrm{Py}\right) .{ }^{13} \mathrm{C}$ NMR $\left(75.8 \mathrm{MHz}, \mathrm{D}_{2} \mathrm{O}\right) \delta$ (ppm): $53.8\left(\mathrm{dt},{ }^{1} J_{\mathrm{CP}}=126.5 \mathrm{~Hz},{ }^{2} J_{\mathrm{CF}}=27.8 \mathrm{~Hz}, \mathrm{CH}\right), 119.1\left(\mathrm{td},{ }^{1} J_{\mathrm{CF}}=247 \mathrm{~Hz},{ }^{2} J_{\mathrm{CP}}=6.0 \mathrm{~Hz}\right.$, $\left.\mathrm{CF}_{2}\right), 121.4\left(\mathrm{~s}, \mathrm{C}_{\mathrm{Py}}\right), 126.3\left(\mathrm{~s}, \mathrm{C}_{\mathrm{Py}}\right), 138.7\left(\mathrm{~s}, \mathrm{C}_{\mathrm{Py}}\right), 149.3\left(\mathrm{~s}, \mathrm{C}_{\mathrm{Py}}\right), 151.3\left(\mathrm{t},{ }^{2} J_{\mathrm{CF}}=27.5 \mathrm{~Hz}\right.$, $\left.\mathrm{CCF}_{2}\right) \cdot{ }^{19} \mathrm{~F}$ NMR $\left(470.3 \mathrm{MHz}, \mathrm{D}_{2} \mathrm{O}\right) \delta(\mathrm{ppm}):-93.6\left(\mathrm{~d},{ }^{2} J_{\mathrm{FAFB}}=257 \mathrm{~Hz}\right),-110.0(\mathrm{dd}$, $\left.{ }^{2} J_{\mathrm{FBFA}}=257 \mathrm{~Hz},{ }^{3} \mathrm{~J}=21.5 \mathrm{~Hz}\right) .{ }^{31} \mathrm{P}$ NMR $\left(202.3 \mathrm{MHz}, \mathrm{D}_{2} \mathrm{O}\right) \delta(\mathrm{ppm})$ : 3.9. Anal. Calc. for $\mathrm{C}_{7} \mathrm{H}_{9} \mathrm{~F}_{2} \mathrm{~N}_{2} \mathrm{O}_{3} \mathrm{P}: \mathrm{C}, 35.31 ; \mathrm{H}, 3.81 ; \mathrm{N}, 11.76$. Found: $\mathrm{C}, 35.13 ; \mathrm{H}, 3.66 ; \mathrm{N}, 11.55$.

\subsection{2. (1-Amino-2,2-difluoro-2-(pyridin-3-yl)ethyl)phosphonic Acid 8b}

Yield $0.15 \mathrm{~g}(62 \%)$; white powder; $\mathrm{mp} 210-125^{\circ} \mathrm{C}$ (decomp.). ${ }^{1} \mathrm{H}$ NMR $(499.8 \mathrm{MHz}$, $\left.\mathrm{D}_{2} \mathrm{O}\right) \delta(\mathrm{ppm}): 3.62(\mathrm{~m}, 1 \mathrm{H}, \mathrm{CH}), 7.66\left(\mathrm{dd},{ }^{3} J_{\mathrm{HH}}=8.2 \mathrm{~Hz},{ }^{3} J_{\mathrm{HH}}=5.1 \mathrm{~Hz}, 1 \mathrm{H}, \mathrm{Py}\right), 8.18(\mathrm{~d}$, $\left.{ }^{3} J_{\mathrm{HH}}=8.2 \mathrm{~Hz}, 1 \mathrm{H}, \mathrm{Py}\right), 8.72\left(\mathrm{~d},{ }^{3} J_{\mathrm{HH}}=5.1 \mathrm{~Hz}, 1 \mathrm{H}, \mathrm{Py}\right), 8.83(\mathrm{~s}, 1 \mathrm{H}, \mathrm{Py}) .{ }^{13} \mathrm{C} \mathrm{NMR}(75.8 \mathrm{MHz}$, $\left.\mathrm{D}_{2} \mathrm{O}\right) \delta(\mathrm{ppm}): 54.8\left(\mathrm{dt},{ }^{1} J_{\mathrm{CP}}=132.7 \mathrm{~Hz},{ }^{2} J_{\mathrm{CF}}=24.1 \mathrm{~Hz}, \mathrm{CH}\right), 119.3\left(\mathrm{t},{ }^{1} J_{\mathrm{CF}}=248 \mathrm{~Hz}, \mathrm{CF}_{2}\right)$, $124.5\left(\mathrm{~s}, \mathrm{C}_{\mathrm{Py}}\right), 130.2\left(\mathrm{td},{ }^{2} J_{\mathrm{CF}}=26 \mathrm{~Hz},{ }^{3} J_{\mathrm{CP}}=4.6 \mathrm{~Hz}, \mathrm{CCF}_{2}\right), 136.3\left(\mathrm{t},{ }^{3} J_{\mathrm{CF}}=5.7 \mathrm{~Hz}, \mathrm{C}_{\mathrm{Py}}\right)$, $145.6\left(\mathrm{t},{ }^{3} J_{\mathrm{CF}}=7.9 \mathrm{~Hz}, \mathrm{C}_{\mathrm{Py}}\right), 150.4\left(\mathrm{~s}, \mathrm{C}_{\mathrm{Py}}\right) .{ }^{19} \mathrm{~F} \mathrm{NMR}\left(470.3 \mathrm{MHz}, \mathrm{D}_{2} \mathrm{O}\right) \delta(\mathrm{ppm}):-86.1(\mathrm{dd}$, $\left.{ }^{2} J_{\mathrm{FAFB}}=253 \mathrm{~Hz},{ }^{3} \mathrm{~J}=24.7 \mathrm{~Hz}\right),-110.1\left(\mathrm{dd},{ }^{2} J_{\mathrm{FBFA}}=253 \mathrm{~Hz},{ }^{3} \mathrm{~J}=24.5 \mathrm{~Hz}\right) .{ }^{31} \mathrm{P}$ NMR $(202.3$ $\left.\mathrm{MHz}, \mathrm{D}_{2} \mathrm{O}\right) \delta(\mathrm{ppm}): 2.8\left(\mathrm{~d},{ }^{2} J_{\mathrm{PH}}=24.3 \mathrm{~Hz}\right)$. Anal. Calc. for $\mathrm{C}_{7} \mathrm{H}_{9} \mathrm{~F}_{2} \mathrm{~N}_{2} \mathrm{O}_{3} \mathrm{P}: \mathrm{C}, 35.31 ; \mathrm{H}$, 3.81; N, 11.76. Found: C, 35.04; H, 3.72; N, 11.59.

\subsection{3. (1-Amino-2,2-difluoro-2-(pyridin-4-yl)ethyl)phosphonic Acid 8c}

Yield $0.16 \mathrm{~g}$ (67\%); white powder; mp $218-220{ }^{\circ} \mathrm{C}$ (decomp.). ${ }^{1} \mathrm{H}$ NMR $(499.8 \mathrm{MHz}$, $\left.\mathrm{D}_{2} \mathrm{O}\right) \delta(\mathrm{ppm}): 4.08(\mathrm{~m}, 1 \mathrm{H}, \mathrm{CH}), 7.73\left(\mathrm{~d},{ }^{3} J_{\mathrm{HH}}=5.3 \mathrm{~Hz}, 2 \mathrm{H}, \mathrm{Py}\right), 8.72\left(\mathrm{~d},{ }^{3} J_{\mathrm{HH}}=5.3 \mathrm{~Hz}\right.$, $2 \mathrm{H}, \mathrm{Py}) .{ }^{13} \mathrm{C}$ NMR $\left(75.8 \mathrm{MHz}, \mathrm{D}_{2} \mathrm{O}\right) \delta(\mathrm{ppm}): 54.3\left(\mathrm{ddd},{ }^{1} J_{\mathrm{CP}}=129.6 \mathrm{~Hz},{ }^{2} J_{\mathrm{CFA}}=27.6 \mathrm{~Hz}\right.$, $\left.{ }^{2} J_{\mathrm{CFB}}=20.1 \mathrm{~Hz}, \mathrm{CH}\right), 119.0\left(\mathrm{td},{ }^{1} J_{\mathrm{CF}}=247.3 \mathrm{~Hz},{ }^{2} J_{\mathrm{CP}}=2.8 \mathrm{~Hz}, \mathrm{CF}_{2}\right), 121.4\left(\mathrm{dd},{ }^{3} J_{\mathrm{CFA}}=7.3 \mathrm{~Hz}\right.$, $\left.{ }^{3} J_{\mathrm{CFB}}=4.8 \mathrm{~Hz}, \mathrm{C}_{\mathrm{Py}}\right), 143.3\left(\mathrm{t},{ }^{2} J_{\mathrm{CF}}=26.2 \mathrm{~Hz}, \underline{C C F}_{2}\right), 148.8\left(\mathrm{~s}, \mathrm{C}_{\mathrm{Py}}\right) .{ }^{19} \mathrm{~F} \mathrm{NMR}(470.3 \mathrm{MHz}$, $\left.\mathrm{D}_{2} \mathrm{O}\right) \delta(\mathrm{ppm}):-89.8\left(\mathrm{~d},{ }^{2} J_{\mathrm{FAFB}}=250 \mathrm{~Hz}\right),-110.2\left(\mathrm{dd},{ }^{2} J_{\mathrm{FBFA}}=250 \mathrm{~Hz},{ }^{3} \mathrm{~J}=23.3 \mathrm{~Hz}\right) \cdot{ }^{31} \mathrm{P}$ NMR (202.3 MHz, $\left.\mathrm{D}_{2} \mathrm{O}\right) \delta(\mathrm{ppm})$ : 3.3. Anal. Calc. for $\mathrm{C}_{7} \mathrm{H}_{9} \mathrm{~F}_{2} \mathrm{~N}_{2} \mathrm{O}_{3} \mathrm{P}: \mathrm{C}, 35.31 ; \mathrm{H}, 3.81 ; \mathrm{N}$, 11.76. Found: $\mathrm{C}, 35.12 ; \mathrm{H}, 3.77 ; \mathrm{N}, 11.63$. 


\subsection{Diethyl (1-Amino-2,2-difluoro-2-(piperidin-4-yl)ethyl)phosphonate 9}

To a solution of aminophosphonate $7 \mathrm{c}(0.3 \mathrm{~g}, 1.02 \mathrm{mmol})$ in $\mathrm{MeOH}(10 \mathrm{~mL})$ was added $\mathrm{Rh} / \mathrm{Al}_{2} \mathrm{O}_{3}(0.1 \mathrm{~g}, 10 \%)$, the mixture was hydrogenated under $50 \mathrm{~atm}$. of $\mathrm{H}_{2}$ for $10 \mathrm{~h}$. After completion of the reaction, the catalyst was filtered off, the solvent was evaporated to give $0.31 \mathrm{~g}$ of 9 (yield $100 \%$ ) as colorless oil. ${ }^{1} \mathrm{H}$ NMR (302 MHz, DMSO-d 6 ) $\delta(\mathrm{ppm})$ : 1.09-1.29 (m, 6H, $\left.\mathrm{CH}_{3}\right), 1.30-1.93\left(\mathrm{~m}, 4 \mathrm{H}, \mathrm{CH}_{2}\right), 2.69-2.85\left(\mathrm{~m}, 2 \mathrm{H}, \mathrm{CH}_{2}\right), 2.87-3.07(\mathrm{~m}$, $1 \mathrm{H}), 3.15-3.30\left(\mathrm{~m}, 2 \mathrm{H}, \mathrm{CH}_{2}\right), 3.47(\mathrm{~m}, 1 \mathrm{H}, \mathrm{CHP}), 3.93-4.17\left(\mathrm{~m}, 4 \mathrm{H}, \mathrm{OCH}_{2}\right), 8.39(\mathrm{~s}, 1 \mathrm{H}$, $\mathrm{NH}) .{ }^{13} \mathrm{C}$ NMR $\left(75.8 \mathrm{MHz}, \mathrm{DMSO}-\mathrm{d}_{6}\right) \delta(\mathrm{ppm}): 16.1\left(\mathrm{~d},{ }^{3} J_{\mathrm{CP}}=5.2 \mathrm{~Hz}, \mathrm{CH}_{3}\right), 16.2(\mathrm{~d}$, $\left.{ }^{3} J_{\mathrm{CP}}=5.2 \mathrm{~Hz}, \mathrm{CH}_{3}\right), 21.4\left(\mathrm{t},{ }^{3} J_{\mathrm{CF}}=4.1 \mathrm{~Hz}, \mathrm{CF}_{2} \mathrm{CHCH}_{2}\right), 22.0\left(\mathrm{t},{ }^{3} J_{\mathrm{CF}}=4.7 \mathrm{~Hz}, \mathrm{CF}_{2} \mathrm{CHCH}_{2}\right)$, $37.3\left(\mathrm{td},{ }^{2} J_{\mathrm{CF}}=23.7 \mathrm{~Hz},{ }^{3} J_{\mathrm{CP}}=4.2 \mathrm{~Hz}, \mathrm{CHCF}_{2}\right), 42.8\left(\mathrm{~s}, 2 \mathrm{CH}_{2}\right), 50.7\left(\mathrm{dt},{ }^{1} J_{\mathrm{CP}}=147.3 \mathrm{~Hz}\right.$, $\left.{ }^{2} J_{\mathrm{CF}}=28.4 \mathrm{~Hz}, \mathrm{CHP}\right), 61.8\left(\mathrm{~d},{ }^{2} J_{\mathrm{CP}}=6.8 \mathrm{~Hz}, \mathrm{OCH}_{2}\right), 62.1\left(\mathrm{~d},{ }^{2} J_{\mathrm{CP}}=6.7 \mathrm{~Hz}, \mathrm{OCH}_{2}\right)$, $124.0\left(\mathrm{td},{ }^{1} J_{\mathrm{CF}}=248 \mathrm{~Hz},{ }^{2} J_{\mathrm{CP}}=30 \mathrm{~Hz}, \mathrm{CF}_{2}\right) .{ }^{19} \mathrm{~F}$ NMR $\left(470.3 \mathrm{MHz}, \mathrm{DMSO}-\mathrm{d}_{6}\right) \delta(\mathrm{ppm}):$ $-111.2(\mathrm{~m}),-111.5(\mathrm{~m}) .{ }^{31} \mathrm{P}$ NMR (202.3 MHz, DMSO-d 6 ) $\delta$ (ppm): 21.3. Anal. Calc. for $\mathrm{C}_{11} \mathrm{H}_{23} \mathrm{~F}_{2} \mathrm{~N}_{2} \mathrm{O}_{3} \mathrm{P}: \mathrm{C}, 44.00 ; \mathrm{H}, 7.72 ; \mathrm{N}$, 9.33. Found: $\mathrm{C}, 44.21 ; \mathrm{H}, 7.65 ; \mathrm{N}, 9.28$.

\section{6. (1-Amino-2,2-difluoro-2-(piperidin-4-yl)ethyl)phosphonic Acid $\mathbf{1 0}$}

To aminophosphonate $9(0.29 \mathrm{~g}, 0.97 \mathrm{mmol})$ was added concentrated $\mathrm{HCl}(1 \mathrm{~mL})$, the reaction mixture was heated at $110{ }^{\circ} \mathrm{C}$ for $2.5 \mathrm{~h}$. After completion of reaction $\mathrm{HCl} / \mathrm{H}_{2} \mathrm{O}$ was evaporated, the residue was dissolved in $\mathrm{MeOH}(1 \mathrm{~mL})$, and treated with propylene oxide $(0.23 \mathrm{~g}, 4 \mathrm{mmol})$. After $24 \mathrm{~h}$ the precipitate formed was separated by filtration, washed with $\mathrm{MeOH}(2 \mathrm{~mL})$ and dried to give $0.17 \mathrm{~g}$ of $\mathbf{1 0}$ (yield $71 \%$ ) as white powder; $\mathrm{mp} 250{ }^{\circ} \mathrm{C}$ (decomp.). ${ }^{1} \mathrm{H}$ NMR $\left(499.8 \mathrm{MHz}, \mathrm{D}_{2} \mathrm{O}\right) \delta(\mathrm{ppm}): 1.65-1.91(\mathrm{~m}, 4 \mathrm{H}), 2.19\left(\mathrm{t},{ }^{2} J_{\mathrm{HH}}=14.9 \mathrm{~Hz}\right.$, $2 \mathrm{H}), 3.07\left(\mathrm{t},{ }^{2} \mathrm{~J}_{\mathrm{HH}}=14.1 \mathrm{~Hz}, 2 \mathrm{H}\right), 3.48-3.58(\mathrm{~m}, 2 \mathrm{H}), 3.70-3.78(\mathrm{~m}, 1 \mathrm{H}, \mathrm{CH}) .{ }^{13} \mathrm{C}$ NMR $\left(150.8 \mathrm{MHz}, \mathrm{D}_{2} \mathrm{O}\right) \delta(\mathrm{ppm}): 27.4\left(\mathrm{~s}, \mathrm{CH}_{2}\right), 28.3\left(\mathrm{~s}, \mathrm{CH}_{2}\right), 37.4\left(\mathrm{t},{ }^{2} J_{\mathrm{CF}}=22.4 \mathrm{~Hz}, \underline{\mathrm{CHCF}}_{2}\right), 42.8$ $\left(\mathrm{s}, \mathrm{CH}_{2}\right), 43.1\left(\mathrm{~s}, \mathrm{CH}_{2}\right), 52.0\left(\mathrm{~d},{ }^{1} J_{\mathrm{CP}}=125.6 \mathrm{~Hz}, \mathrm{CH}\right), 122.5\left(\mathrm{t},{ }^{1} J_{\mathrm{CF}}=248 \mathrm{~Hz}, \mathrm{CF}_{2}\right) \cdot{ }^{19} \mathrm{~F}$ NMR $\left(470.3 \mathrm{MHz}, \mathrm{D}_{2} \mathrm{O}\right) \delta(\mathrm{ppm}):-107.3\left(\mathrm{ddd},{ }^{2} J_{\mathrm{FAFB}}=246 \mathrm{~Hz},{ }^{3} J_{\mathrm{FH}}=26.5 \mathrm{~Hz},{ }^{3} J_{\mathrm{FP}}=26.5 \mathrm{~Hz}\right)$, $-111.6\left(\mathrm{dd},{ }^{2} J_{\mathrm{FBFA}}=246 \mathrm{~Hz},{ }^{3} \mathrm{~J}=26.5 \mathrm{~Hz}\right) .{ }^{31} \mathrm{P} \mathrm{NMR}\left(202.3 \mathrm{MHz}, \mathrm{D}_{2} \mathrm{O}\right) \delta(\mathrm{ppm}): 3.5$. Anal. Calc. for $\mathrm{C}_{7} \mathrm{H}_{15} \mathrm{~F}_{2} \mathrm{~N}_{2} \mathrm{O}_{3} \mathrm{P}: \mathrm{C}, 34.43 ; \mathrm{H}, 6.19 ; \mathrm{N}, 11.47$. Found: $\mathrm{C}, 34.17 ; \mathrm{H}, 6.07 ; \mathrm{N}, 11.63$.

4.7. Synthesis of Diethyl (2-(Difluoro(pyridinyl)methyl)-4-oxothiazolidin-2-yl)phosphonates 11a-c: General Procedure

To solution of respective iminophosphonate $2(0.12 \mathrm{~g}, 0.41 \mathrm{mmol})$ in benzene $(1 \mathrm{~mL})$ was added mercaptoacetic acid $(0.04 \mathrm{~g}, 0.41 \mathrm{mmol})$. The reaction mixture was refluxed for $2 \mathrm{~h}$, the solvent was evaporated in vacuum and the residue was triturated with hexane.

\subsubsection{Diethyl (2-(Difluoro(pyridin-2-yl)methyl)-4-oxothiazolidin-2-yl)phosphonate 11a}

Yield $0.11 \mathrm{~g}(73 \%)$; yellow oil. ${ }^{1} \mathrm{H}$ NMR $\left(302 \mathrm{MHz}, \mathrm{CDCl}_{3}\right) \delta(\mathrm{ppm}): 1.11-1.30(\mathrm{~m}$, $\left.{ }^{3} J_{\mathrm{HH}}=7.1 \mathrm{~Hz}, 6 \mathrm{H}, \mathrm{CH}_{3}\right), 3.44\left(\mathrm{dd},{ }^{2} J_{\mathrm{HAHB}}=15.1 \mathrm{~Hz},{ }^{4} J_{\mathrm{HAP}}=5.8 \mathrm{~Hz}, 1 \mathrm{H}, \mathrm{SCH}_{\mathrm{A}} \mathrm{H}_{\mathrm{B}}\right), 3.6$ $\left(\mathrm{d},{ }^{2} J_{\mathrm{HBHA}}=15.1 \mathrm{~Hz}, 1 \mathrm{H}, \mathrm{SCH}_{\mathrm{A}} \mathrm{H}_{\mathrm{B}}\right), 3.93-4.22\left(\mathrm{~m}, 4 \mathrm{H}, \mathrm{OCH}_{2}\right), 7.45\left(\mathrm{dd},{ }^{3} J_{\mathrm{HH}}=7.9 \mathrm{~Hz}\right.$, $\left.{ }^{3} J_{\mathrm{HH}}=4.9 \mathrm{~Hz}, 1 \mathrm{H}, \mathrm{Py}\right), 7.74\left(\mathrm{~d},{ }^{3} J_{\mathrm{HH}}=7.9 \mathrm{~Hz}, 1 \mathrm{H}, \mathrm{Py}\right), 7.86\left(\mathrm{t},{ }^{3} J_{\mathrm{HH}}=7.9 \mathrm{~Hz}, 1 \mathrm{H}, \mathrm{Py}\right)$, $8.20(\mathrm{~s}, 1 \mathrm{H}, \mathrm{NH}), 8.65\left(\mathrm{~d},{ }^{3} J_{\mathrm{HH}}=4.9 \mathrm{~Hz}, 1 \mathrm{H}, \mathrm{Py}\right) .{ }^{13} \mathrm{C} \mathrm{NMR}\left(150.8 \mathrm{MHz}, \mathrm{CDCl}_{3}\right) \delta$ (ppm): $16.3\left(\mathrm{~d},{ }^{3} J_{\mathrm{CP}}=5.9 \mathrm{~Hz}, \mathrm{CH}_{3}\right), 16.4\left(\mathrm{~d},{ }^{3} J_{\mathrm{CP}}=5.6 \mathrm{~Hz}, \mathrm{CH}_{3}\right), 32.1\left(\mathrm{~s}, \mathrm{SCH}_{2}\right), 64.8(\mathrm{~d}$, $\left.{ }^{2} J_{\mathrm{CP}}=7.7 \mathrm{~Hz}, \mathrm{OCH}_{2}\right), 65.2\left(\mathrm{~d},{ }^{2} J_{\mathrm{CP}}=7.6 \mathrm{~Hz}, \mathrm{OCH}_{2}\right), 68.5\left(\mathrm{dt},{ }^{1} J_{\mathrm{CP}}=170.7 \mathrm{~Hz},{ }^{2} J_{\mathrm{CF}}=32.6 \mathrm{~Hz}\right.$, $\mathrm{CP}), 116.0\left(\mathrm{td},{ }^{1} J_{\mathrm{CF}}=254 \mathrm{~Hz},{ }^{2} J_{\mathrm{CP}}=21 \mathrm{~Hz}, \mathrm{CF}_{2}\right), 121.7\left(\mathrm{t},{ }^{3} J_{\mathrm{CF}}=4.5 \mathrm{~Hz}, \mathrm{C}_{\mathrm{Py}}\right), 125.6(\mathrm{~s}$, $\left.\mathrm{C}_{\mathrm{Py}}\right), 137.5\left(\mathrm{~s}, \mathrm{C}_{\mathrm{Py}}\right), 149.0\left(\mathrm{~s}, \mathrm{C}_{\mathrm{Py}}\right), 152.2\left(\mathrm{t},{ }^{2} J_{\mathrm{CF}}=30 \mathrm{~Hz}, \mathrm{CCF}_{2}\right), 174.1\left(\mathrm{~d},{ }^{3} J_{\mathrm{CP}}=5.2 \mathrm{~Hz}\right.$, $\mathrm{C}(\mathrm{O})) .{ }^{19} \mathrm{~F} \mathrm{NMR}\left(470.3 \mathrm{MHz}, \mathrm{CDCl}_{3}\right) \delta(\mathrm{ppm}):-98.5\left(\mathrm{dd},{ }^{2} J_{\mathrm{FAFB}}=260 \mathrm{~Hz},{ }^{3} J_{\mathrm{FP}}=12 \mathrm{~Hz}\right)$, $-108.4\left(\mathrm{~d}^{2} J_{\mathrm{FBFA}}=260 \mathrm{~Hz}\right) .{ }^{31} \mathrm{P} \mathrm{NMR}\left(202.3 \mathrm{MHz}, \mathrm{CDCl}_{3}\right) \delta(\mathrm{ppm})$ :13.5. Anal. Calc. for $\mathrm{C}_{13} \mathrm{H}_{17} \mathrm{~F}_{2} \mathrm{~N}_{2} \mathrm{O}_{4} \mathrm{PS}: \mathrm{C}, 42.62 ; \mathrm{H}, 4.68 ; \mathrm{N}, 7.65 ; \mathrm{S}, 8.75$. Found: $\mathrm{C}, 42.44 ; \mathrm{H}, 4.53 ; \mathrm{N}, 7.51 ; \mathrm{S}, 8.62$.

\subsubsection{Diethyl (2-(Difluoro(pyridin-3-yl)methyl)-4-oxothiazolidin-2-yl)phosphonate 11b}

Yield $0.125 \mathrm{~g}(83 \%)$; white powder; $\mathrm{mp} 162-164{ }^{\circ} \mathrm{C} .{ }^{1} \mathrm{H} \mathrm{NMR}\left(302 \mathrm{MHz}, \mathrm{CDCl}_{3}\right) \delta$ (ppm): $1.30\left(\mathrm{t}^{3}{ }^{3} \mathrm{HH}_{\mathrm{HH}}=6.8 \mathrm{~Hz}, 6 \mathrm{H}, \mathrm{CH}_{3}\right), 3.05\left(\mathrm{dd},{ }^{2} J_{\mathrm{HAHB}}=15.3 \mathrm{~Hz},{ }^{4} J_{\mathrm{HAP}}=5.9 \mathrm{~Hz}, 1 \mathrm{H}\right.$, $\left.\mathrm{SCH}_{\mathrm{A}} \mathrm{H}_{\mathrm{B}}\right), 3.39\left(\mathrm{~d},{ }^{2} J_{\mathrm{HBHA}}=15.3 \mathrm{~Hz}, 1 \mathrm{H}, \mathrm{SCH}_{\mathrm{A}} \underline{\mathrm{H}_{\mathrm{B}}}\right), 4.09-4.45\left(\mathrm{~m}, 4 \mathrm{H}, \mathrm{OCH}_{2}\right), 7.38(\mathrm{dd}$, $\left.{ }^{3} J_{\mathrm{HH}}=8.0 \mathrm{~Hz},{ }^{3} J_{\mathrm{HH}}=4.9 \mathrm{~Hz}, 1 \mathrm{H}, \mathrm{Py}\right), 7.99\left(\mathrm{~d},{ }^{3} J_{\mathrm{HH}}=8.0 \mathrm{~Hz}, 1 \mathrm{H}, \mathrm{Py}\right), 8.73\left(\mathrm{~d},{ }^{3} J_{\mathrm{HH}}=4.9 \mathrm{~Hz}\right.$, 
$1 \mathrm{H}, \mathrm{Py}), 8.73$ (s, 1H, NH), 8.91 (s, 1H, Py). $\left.{ }^{13} \mathrm{C} \mathrm{NMR} \mathrm{(150.8} \mathrm{MHz,} \mathrm{CDCl}_{3}\right) \delta(\mathrm{ppm}): 16.3$ $\left(\mathrm{d},{ }^{3} J_{\mathrm{CP}}=6 \mathrm{~Hz}, \mathrm{CH}_{3}\right), 16.4\left(\mathrm{~d},{ }^{3} J_{\mathrm{CP}}=6 \mathrm{~Hz}, \mathrm{CH}_{3}\right), 32.1\left(\mathrm{~s}, \mathrm{SCH}_{2}\right), 65.0\left(\mathrm{~d},{ }^{2} J_{\mathrm{CP}}=8 \mathrm{~Hz}\right.$, $\left.\mathrm{OCH}_{2}\right), 65.1\left(\mathrm{~d},{ }^{2} J_{\mathrm{CP}}=8 \mathrm{~Hz}, \mathrm{OCH}_{2}\right), 68.0\left(\mathrm{dt},{ }^{1} J_{\mathrm{CP}}=167 \mathrm{~Hz},{ }^{2} J_{\mathrm{CF}}=32 \mathrm{~Hz}, \mathrm{CP}\right), 120.7$ $\left(\mathrm{td},{ }^{1} J_{\mathrm{CF}}=255 \mathrm{~Hz},{ }^{2} J_{\mathrm{CP}}=12 \mathrm{~Hz}, \mathrm{CF}_{2}\right), 122.7\left(\mathrm{~s}, \mathrm{C}_{\mathrm{Py}}\right), 128.2\left(\mathrm{td},{ }^{2} J_{\mathrm{CF}}=27 \mathrm{~Hz},{ }^{3} J_{\mathrm{CP}}=4 \mathrm{~Hz}\right.$, $\left.\mathrm{CCF}_{2}\right), 136.0\left(\mathrm{t},{ }^{3} J_{\mathrm{CF}}=7 \mathrm{~Hz}, \mathrm{C}_{\mathrm{Py}}\right), 148.6\left(\mathrm{t},{ }^{3} J_{\mathrm{CF}}=5 \mathrm{~Hz}, \mathrm{C}_{\mathrm{Py}}\right), 151.4\left(\mathrm{~s}, \mathrm{C}_{\mathrm{Py}}\right), 174.8(\mathrm{~d}$, $\left.{ }^{3} J_{\mathrm{CP}}=7 \mathrm{~Hz}, \mathrm{C}(\mathrm{O})\right) .{ }^{19} \mathrm{~F} \mathrm{NMR}\left(188 \mathrm{MHz}, \mathrm{CDCl}_{3}\right) \delta(\mathrm{ppm}):-96.7\left(\mathrm{~d},{ }^{2} J_{\mathrm{FAFB}}=255.5 \mathrm{~Hz}\right)$, $-101.9\left(\mathrm{~d},{ }^{2} J_{\mathrm{FBFA}}=255.5 \mathrm{~Hz}\right) .{ }^{31} \mathrm{P}$ NMR $\left(202.3 \mathrm{MHz}, \mathrm{CDCl}_{3}\right) \delta(\mathrm{ppm}): 13.4$. Anal. Calc. for $\mathrm{C}_{13} \mathrm{H}_{17} \mathrm{~F}_{2} \mathrm{~N}_{2} \mathrm{O}_{4} \mathrm{PS}: \mathrm{C}, 42.62 ; \mathrm{H}, 4.68 ; \mathrm{N}, 7.65 ; \mathrm{S}, 8.75$. Found: $\mathrm{C}, 42.47 ; \mathrm{H}, 4.73 ; \mathrm{N}, 7.41 ; \mathrm{S}, 8.58$

\subsubsection{Diethyl (2-(Difluoro(pyridin-4-yl)methyl)-4-oxothiazolidin-2-yl)phosphonate 11c}

Yield $0.116 \mathrm{~g}(77 \%)$; white powder; mp $80-82{ }^{\circ} \mathrm{C} .{ }^{1} \mathrm{H}$ NMR $\left(499.8 \mathrm{MHz}, \mathrm{CDCl}_{3}\right)$ $\delta(\mathrm{ppm}): 1.31\left(\mathrm{t},{ }^{3} J_{\mathrm{HH}}=7 \mathrm{~Hz}, 6 \mathrm{H}, \mathrm{CH}_{3}\right), 3.1\left(\mathrm{dd},{ }^{2} J_{\mathrm{HAHB}}=15.2 \mathrm{~Hz},{ }^{4} J_{\mathrm{HAP}}=5.6 \mathrm{~Hz}\right.$, $\left.1 \mathrm{H}, \mathrm{SCH}_{\mathrm{A}} \mathrm{H}_{\mathrm{B}}\right), 3.4\left(\mathrm{~d},{ }^{2} J_{\mathrm{HBHA}}=15.2 \mathrm{~Hz}, 1 \mathrm{H}, \mathrm{SCH}_{\mathrm{A}} \mathrm{H}_{\mathrm{B}}\right), 4.12-4.33\left(\mathrm{~m}, 4 \mathrm{H}, \mathrm{OCH}_{2}\right), 7.58$ $\left(\mathrm{d},{ }^{3} J_{\mathrm{HH}}=5.3 \mathrm{~Hz}, 2 \mathrm{H}, \mathrm{Py}\right), 8.26(\mathrm{~s}, 1 \mathrm{H}, \mathrm{NH}), 8.73\left(\mathrm{~d},{ }^{3} \mathrm{JHH}_{\mathrm{HH}}=5.3 \mathrm{~Hz}, 2 \mathrm{H}, \mathrm{Py}\right) \cdot{ }^{13} \mathrm{C}$ NMR $\left(150.8 \mathrm{MHz}, \mathrm{CDCl}_{3}\right) \delta(\mathrm{ppm}): 16.3\left(\mathrm{~d},{ }^{3} J_{\mathrm{CP}}=5.7 \mathrm{~Hz}, \mathrm{CH}_{3}\right), 16.4\left(\mathrm{~d},{ }^{3} J_{\mathrm{CP}}=5.7 \mathrm{~Hz}, \mathrm{CH}_{3}\right)$, $32.1\left(\mathrm{~s}, \mathrm{SCH}_{2}\right), 65.0\left(\mathrm{~d},{ }^{2} J_{\mathrm{CP}}=7.3 \mathrm{~Hz}, \mathrm{OCH}_{2}\right), 65.2\left(\mathrm{~d},{ }^{2} J_{\mathrm{CP}}=7.3 \mathrm{~Hz}, \mathrm{OCH}_{2}\right), 67.6(\mathrm{dt}$, $\left.{ }^{1} J_{\mathrm{CP}}=167.1 \mathrm{~Hz},{ }^{2} J_{\mathrm{CF}}=34.2 \mathrm{~Hz}, \mathrm{CP}\right), 120.2\left(\mathrm{td},{ }^{1} J_{\mathrm{CF}}=253 \mathrm{~Hz},{ }^{2} J_{\mathrm{CP}}=11.6 \mathrm{~Hz}, \mathrm{CF}_{2}\right), 122.4(\mathrm{t}$,

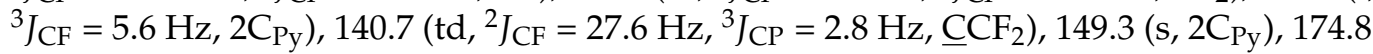
$\left(\mathrm{d},{ }^{3} J_{\mathrm{CP}}=6.5 \mathrm{~Hz}, \mathrm{CO}\right) .{ }^{19} \mathrm{~F} \mathrm{NMR}\left(470.3 \mathrm{MHz}, \mathrm{CDCl}_{3}\right) \delta(\mathrm{ppm}):-98.9\left(\mathrm{~d},{ }^{2} J_{\mathrm{FAFB}}=252 \mathrm{~Hz}\right)$, $-104.0\left(\mathrm{~d}^{2} \mathrm{~J}_{\mathrm{FBFA}}=252 \mathrm{~Hz}\right) .{ }^{31} \mathrm{P}$ NMR $\left(202.3 \mathrm{MHz}, \mathrm{CDCl}_{3}\right) \delta(\mathrm{ppm})$ : 13.2.Anal. Calc. for $\mathrm{C}_{13} \mathrm{H}_{17} \mathrm{~F}_{2} \mathrm{~N}_{2} \mathrm{O}_{4} \mathrm{PS}: \mathrm{C}, 42.62 ; \mathrm{H}, 4.68 ; \mathrm{N}, 7.65 ; \mathrm{S}, 8.75$. Found: $\mathrm{C}, 42.39 ; \mathrm{H}, 4.56 ; \mathrm{N}, 7.48 ; \mathrm{S}, 8.64$.

\subsection{Synthesis of Diethyl (2-(Difluoro(pyridinyl)methyl)-4-oxo-1,3-thiazinan-2-yl)phosphonates} 12a-c: General Procedure

To a solution of respective iminophosphonate $2(0.13 \mathrm{~g}, 0.44 \mathrm{mmol})$ in benzene $(1 \mathrm{~mL})$ was added 3-mercaptopropionic acid $(0.05 \mathrm{~g}, 0.44 \mathrm{mmol})$. The reaction mixture was refluxed for $8 \mathrm{~h}$, the solvent was evaporated in vacuum and the residue was triturated with $\mathrm{Et}_{2} \mathrm{O}$.

\subsubsection{Diethyl (2-(Difluoro(pyridine-2-yl)methyl)-4-oxo-1,3-thiazinan-2-yl)phosphonate 12a}

Yield $0.13 \mathrm{~g}(76 \%)$; yellow oil. ${ }^{1} \mathrm{H} \mathrm{NMR}\left(302 \mathrm{MHz}, \mathrm{CDCl}_{3}\right) \delta(\mathrm{ppm}): 1.13\left(\mathrm{t},{ }^{3} \mathrm{~J}_{\mathrm{HH}}=7.1 \mathrm{~Hz}\right.$, $\left.3 \mathrm{H}, \mathrm{CH}_{3}\right), 1.25\left(\mathrm{t},{ }^{3} \mathrm{H}_{\mathrm{HH}}=7.1 \mathrm{~Hz}, 3 \mathrm{H}, \mathrm{CH}_{3}\right), 2.73-2.84\left(\mathrm{~m}, 2 \mathrm{H}, \mathrm{CH}_{2}\right), 2.91-3.01(\mathrm{~m}, 1 \mathrm{H}, \mathrm{CH})$, $3.22-3.35(\mathrm{~m}, 1 \mathrm{H}, \mathrm{CH}), 3.96-4.28\left(\mathrm{~m}, 4 \mathrm{H}, 2 \mathrm{OCH}_{2}\right), 7.46\left(\mathrm{dd},{ }^{3} J_{\mathrm{HH}}=7.9 \mathrm{~Hz},{ }^{3} \mathrm{JHH}_{\mathrm{HH}}=5.1 \mathrm{~Hz}\right.$, $1 \mathrm{H}), 7.70\left(\mathrm{~d},{ }^{3} J_{\mathrm{HH}}=7.9 \mathrm{~Hz}, 1 \mathrm{H}\right), 7.86\left(\mathrm{t},{ }^{3} J_{\mathrm{HH}}=7.9 \mathrm{~Hz}, 1 \mathrm{H}\right), 8.09(\mathrm{~s}, 1 \mathrm{H}, \mathrm{NH}), 8.70(\mathrm{~d}$, $\left.{ }^{3} J_{\mathrm{HH}}=5.1 \mathrm{~Hz}, 1 \mathrm{H}\right) .{ }^{13} \mathrm{C} \mathrm{NMR}\left(125.7 \mathrm{MHz}, \mathrm{CDCl}_{3}\right) \delta(\mathrm{ppm}): 16.2\left(\mathrm{~d},{ }^{3} J_{\mathrm{CP}}=6 \mathrm{~Hz}, \mathrm{CH}_{3}\right)$, $16.3\left(\mathrm{~d},{ }^{3} J_{\mathrm{CP}}=5 \mathrm{~Hz}, \mathrm{CH}_{3}\right), 23.5\left(\mathrm{~s}, \mathrm{SCH}_{2}\right), 33.3\left(\mathrm{~s}, \mathrm{CH}_{2} \mathrm{CO}\right), 64.7\left(\mathrm{~d},{ }^{2} J_{\mathrm{CP}}=7.7 \mathrm{~Hz}, \mathrm{OCH}_{2}\right)$, $65.3\left(\mathrm{~d},{ }^{2} J_{\mathrm{CP}}=7.6 \mathrm{~Hz}, \mathrm{OCH}_{2}\right), 66.6\left(\mathrm{dm},{ }^{1} J_{\mathrm{CP}}=168.7 \mathrm{~Hz}, \mathrm{CP}\right), 116.0\left(\mathrm{td},{ }^{1} J_{\mathrm{CF}}=256.2 \mathrm{~Hz}\right.$, $\left.{ }^{2} J_{\mathrm{CP}}=15 \mathrm{~Hz}, \mathrm{CF}_{2}\right), 122.1\left(\mathrm{~s}, \mathrm{C}_{\mathrm{Py}}\right), 125.5\left(\mathrm{~s}, \mathrm{C}_{\mathrm{Py}}\right), 137.2\left(\mathrm{~s}, \mathrm{C}_{\mathrm{Py}}\right), 148.8\left(\mathrm{~s}, \mathrm{C}_{\mathrm{Py}}\right), 152.2(\mathrm{td}$, $\left.{ }^{2} J_{\mathrm{CF}}=30 \mathrm{~Hz},{ }^{3} J_{\mathrm{CP}}=2.5 \mathrm{~Hz}, \mathrm{CCF}_{2}\right), 170.9\left(\mathrm{~d},{ }^{3} J_{\mathrm{CP}}=3.1 \mathrm{~Hz}, \mathrm{CO}\right) .{ }^{19} \mathrm{~F} \mathrm{NMR}(470.3 \mathrm{MHz}$, $\left.\mathrm{CDCl}_{3}\right) \delta(\mathrm{ppm}):-99\left(\mathrm{dd},{ }^{2} J_{\mathrm{FAFB}}=255 \mathrm{~Hz},{ }^{3} J_{\mathrm{FP}}=9 \mathrm{~Hz}\right),-106.4\left(\mathrm{~d},{ }^{2} J_{\mathrm{FBFA}}=255 \mathrm{~Hz}\right) \cdot{ }^{31} \mathrm{P}$ NMR (202.3 MHz, $\left.\mathrm{CDCl}_{3}\right) \delta(\mathrm{ppm}): 13.2\left(\mathrm{~m},{ }^{3} J=9 \mathrm{~Hz}\right)$. Anal. Calc. for $\mathrm{C}_{14} \mathrm{H}_{19} \mathrm{~F}_{2} \mathrm{~N}_{2} \mathrm{O}_{4} \mathrm{PS}$ : C, 44.21; H, 5.04; N, 7.37; S, 8.42. Found: C, 44.39; H, 4.91; N, 7.43; S, 8.34.

\subsubsection{Diethyl (2-(Difluoro(pyridine-3-yl)methyl)-4-oxo-1,3-thiazinan-2-yl)phosphonate 12b}

Yield $0.14 \mathrm{~g}$ (82\%); white powder; mp $158-160{ }^{\circ} \mathrm{C} .{ }^{1} \mathrm{H} \mathrm{NMR}\left(400 \mathrm{MHz}, \mathrm{CDCl}_{3}\right) \delta$ (ppm): $1.31\left(\mathrm{t},{ }^{3} \mathrm{~J}_{\mathrm{HH}}=7.2 \mathrm{~Hz}, 6 \mathrm{H}, \mathrm{CH}_{3}\right), 2.32-2.44\left(\mathrm{~m}, 2 \mathrm{H}, \mathrm{CH}_{2}\right), 2.53-2.68\left(\mathrm{~m}, 1 \mathrm{H}, \mathrm{CH}_{2}\right)$, $2.80-2.89\left(\mathrm{~m}, 1 \mathrm{H}, \mathrm{CH}_{2}\right), 4.10-4.35\left(\mathrm{~m}, 4 \mathrm{H}, \mathrm{OCH}_{2}\right), 6.72(\mathrm{~s}, 1 \mathrm{H}, \mathrm{NH}), 7.37\left(\mathrm{dd},{ }^{3} J_{\mathrm{HH}}=8.1 \mathrm{~Hz}\right.$, $\left.{ }^{3} J_{\mathrm{HH}}=4.9 \mathrm{~Hz}, 1 \mathrm{H}, \mathrm{Py}\right), 7.92\left(\mathrm{~d},{ }^{3} J_{\mathrm{HH}}=8.1 \mathrm{~Hz}, 1 \mathrm{H}, \mathrm{Py}\right), 8.72\left(\mathrm{~d},{ }^{3} J_{\mathrm{HH}}=4.9 \mathrm{~Hz}, 1 \mathrm{H}, \mathrm{Py}\right), 8.87$ (s, 1H, Py). ${ }^{13} \mathrm{C}$ NMR $\left(150.8 \mathrm{MHz}, \mathrm{CDCl}_{3}\right) \delta(\mathrm{ppm}): 16.40\left(\mathrm{~d},{ }^{3} J_{\mathrm{CP}}=5.7 \mathrm{~Hz}, \mathrm{CH}_{3}\right), 16.43$ $\left(\mathrm{d},{ }^{3} J_{\mathrm{CP}}=5.6 \mathrm{~Hz}, \mathrm{CH}_{3}\right), 23.4\left(\mathrm{~s}, \mathrm{SCH}_{2}\right), 33.4\left(\mathrm{~s}, \mathrm{CH}_{2} \mathrm{CO}\right), 65.0\left(\mathrm{~d},{ }^{2} J_{\mathrm{CP}}=7.5 \mathrm{~Hz}, \mathrm{OCH}_{2}\right)$, $65.2\left(\mathrm{~d},{ }^{2} J_{\mathrm{CP}}=7.5 \mathrm{~Hz}, \mathrm{OCH}_{2}\right), 65.5\left(\mathrm{dt},{ }^{1} J_{\mathrm{CP}}=161.2 \mathrm{~Hz},{ }^{2} J_{\mathrm{CF}}=29.5 \mathrm{~Hz}, \mathrm{CP}\right), 120.5(\mathrm{td}$, $\left.{ }^{1} J_{\mathrm{CF}}=255.7 \mathrm{~Hz},{ }^{2} J_{\mathrm{CP}}=7.4 \mathrm{~Hz}, \mathrm{CF}_{2}\right), 122.5\left(\mathrm{~s}, \mathrm{C}_{\mathrm{Py}}\right), 128.3\left(\mathrm{td},{ }^{2} J_{\mathrm{CF}}=26.7 \mathrm{~Hz},{ }^{3} J_{\mathrm{CP}}=3.6 \mathrm{~Hz}\right.$, $\left.\mathrm{CCF}_{2}\right), 135.9\left(\mathrm{t},{ }^{3} J_{\mathrm{CF}}=5.9 \mathrm{~Hz}, \mathrm{C}_{\mathrm{Py}}\right), 148.9\left(\mathrm{t},{ }^{3} J_{\mathrm{CF}}=6.4 \mathrm{~Hz}, \mathrm{C}_{\mathrm{Py}}\right), 151.5\left(\mathrm{~s}, \mathrm{C}_{\mathrm{Py}}\right), 170.4(\mathrm{~d}$, $\left.{ }^{3} J_{\mathrm{CP}}=3.1 \mathrm{~Hz}, \mathrm{C}(\mathrm{O})\right) .{ }^{19} \mathrm{~F} \mathrm{NMR}\left(470.3 \mathrm{MHz}, \mathrm{CDCl}_{3}\right) \delta(\mathrm{ppm}):-98.8\left(\mathrm{~d},{ }^{2} J_{\mathrm{FAFB}}=253.6 \mathrm{~Hz}\right)$, 
$-102.2\left(\mathrm{~d}^{2} \mathrm{~J}_{\mathrm{FBFA}}=253.6 \mathrm{~Hz}\right) .{ }^{31} \mathrm{P}$ NMR $\left(202.3 \mathrm{MHz}, \mathrm{CDCl}_{3}\right) \delta(\mathrm{ppm}): 13.5$. Anal. Calc. for $\mathrm{C}_{14} \mathrm{H}_{19} \mathrm{~F}_{2} \mathrm{~N}_{2} \mathrm{O}_{4}$ PS: C, 44.21; H, 5.04; N, 7.37; S, 8.42. Found: $\mathrm{C}, 44.08 ; \mathrm{H}, 4.97 ; \mathrm{N}, 7.48 ; \mathrm{S}, 8.31$.

4.8.3. Diethyl (2-(Difluoro(pyridine-4-yl)methyl)-4-oxo-1,3-thiazinan-2-yl)phosphonate 12c

Yield $0.13 \mathrm{~g}(76 \%)$; white powder; $\mathrm{mp} 110{ }^{\circ} \mathrm{C} .{ }^{1} \mathrm{H} \mathrm{NMR}\left(400 \mathrm{MHz}, \mathrm{CDCl}_{3}\right) \delta$ (ppm): $1.27-1.38\left(\mathrm{~m},{ }^{3} \mathrm{~J}_{\mathrm{HH}}=7.4 \mathrm{~Hz}, 6 \mathrm{H}, \mathrm{CH}_{3}\right), 2.41-2.53\left(\mathrm{~m}, 2 \mathrm{H}, \mathrm{CH}_{2}\right), 2.56-2.67(\mathrm{~m}, 1 \mathrm{H}, \mathrm{CH})$, 2.84-2.92 (m, 1H, CH), 4.19-4.31 (m, 4H, OCH $), 6.39(\mathrm{~s}, 1 \mathrm{H}, \mathrm{NH}), 7.65\left(\mathrm{~d},{ }^{3} J_{\mathrm{HH}}=5.1 \mathrm{~Hz}\right.$, $2 \mathrm{H}, \mathrm{Py}), 8.76\left(\mathrm{~d},{ }^{3} \mathrm{~J}_{\mathrm{HH}}=5.1 \mathrm{~Hz}, 2 \mathrm{H}, \mathrm{Py}\right) .{ }^{13} \mathrm{C} \mathrm{NMR}\left(150.8 \mathrm{MHz}, \mathrm{CDCl}_{3}\right) \delta(\mathrm{ppm}): 16.4(\mathrm{br} \mathrm{d}$, $\left.{ }^{3} J_{\mathrm{CP}}=5.7 \mathrm{~Hz}, \mathrm{CH}_{3}\right), 23.4\left(\mathrm{~s}, \mathrm{SCH}_{2}\right), 33.4\left(\mathrm{~s}, \mathrm{CH}_{2} \mathrm{CO}\right), 65.2\left(\mathrm{~d}^{2}{ }^{2} J_{\mathrm{CP}}=7.5 \mathrm{~Hz}, \mathrm{OCH}_{2}\right), 65.2(\mathrm{dt}$, $\left.{ }^{1} J_{\mathrm{CP}}=161.4 \mathrm{~Hz},{ }^{2} J_{\mathrm{CF}}=30.8 \mathrm{~Hz}, \mathrm{CP}\right), 65.3\left(\mathrm{~d},{ }^{2} J_{\mathrm{CP}}=7.5 \mathrm{~Hz}, \mathrm{OCH}_{2}\right), 119.9\left(\mathrm{td},{ }^{1} J_{\mathrm{CF}}=257 \mathrm{~Hz}\right.$, $\left.{ }^{2} J_{\mathrm{CP}}=8 \mathrm{~Hz}, \mathrm{CF}_{2}\right), 122.4\left(\mathrm{t},{ }^{3} J_{\mathrm{CF}}=5.7 \mathrm{~Hz}, 2 \mathrm{C}_{\mathrm{Py}}\right), 140.8\left(\mathrm{td},{ }^{2} J_{\mathrm{CF}}=28.1 \mathrm{~Hz},{ }^{3} J_{\mathrm{CP}}=4.8 \mathrm{~Hz}\right.$, $\left.\mathrm{CCF}_{2}\right), 149.3\left(\mathrm{~s}, 2 \mathrm{C}_{\mathrm{Py}}\right), 170.6\left(\mathrm{~d},{ }^{3} J_{\mathrm{CP}}=2.7 \mathrm{~Hz}, \mathrm{CO}\right) .{ }^{19} \mathrm{~F} \mathrm{NMR}\left(470.3 \mathrm{MHz}, \mathrm{CDCl}_{3}\right) \delta(\mathrm{ppm})$ : $-100.4\left(\mathrm{~d},{ }^{2} J_{\mathrm{FAFB}}=251 \mathrm{~Hz}\right),-103.5\left(\mathrm{~d},{ }^{2} J_{\mathrm{FBFA}}=251 \mathrm{~Hz}\right) \cdot{ }^{31} \mathrm{P} \mathrm{NMR}\left(202.3 \mathrm{MHz}, \mathrm{CDCl}_{3}\right) \delta$ (ppm): 12.9. Anal. Calc. for $\mathrm{C}_{14} \mathrm{H}_{19} \mathrm{~F}_{2} \mathrm{~N}_{2} \mathrm{O}_{4} \mathrm{PS}: \mathrm{C}, 44.21 ; \mathrm{H}, 5.04 ; \mathrm{N}, 7.37 ; \mathrm{S}, 8.42$. Found: $\mathrm{C}$, $44.48 ; \mathrm{H}, 4.88 ; \mathrm{N}, 7.28 ; \mathrm{S}, 8.28$.

Supplementary Materials: The following are available online at https:/ /www.mdpi.com/article/10 $.3390 / \mathrm{org} 2020007 / \mathrm{s} 1,{ }^{1} \mathrm{H},{ }^{19} \mathrm{~F}$, and ${ }^{13} \mathrm{C}$ NMR spectra of compounds synthesized.

Author Contributions: Conceptualization, P.P.O. and Y.V.R.; methodology, P.P.O., Y.V.R., O.M.S., L.V.B. and A.V.B.; validation, P.P.O., Y.V.R., O.M.S. and L.V.B.; investigation, O.M.S., L.V.B. and A.V.B.; data curation, P.P.O. and Y.V.R.; writing - original draft preparation, P.P.O. and Y.V.R.; writingreview and editing, P.P.O., Y.V.R. and O.M.S. All authors have read and agreed to the published version of the manuscript.

Funding: This research received no external funding.

Institutional Review Board Statement: Not applicable.

Informed Consent Statement: Not applicable.

Data Availability Statement: Data is contained within the article and supplementary material.

Conflicts of Interest: The authors declare no conflict of interest.

\section{References}

1. Kukhar, V.P.; Hudson, H.R. Aminophosphonic and Aminophosphinic Acids. Chemistry and Biological Activity; John Wiley \& Sons: New York, NY, USA, 2000.

2. Kafarski, P.; Lejczak, B. Biological activity of aminophosphonic acids. Phosphorus Sulfur Silicon Relat. Elem. 1991, 63, $193-215$. [CrossRef]

3. Mucha, A.; Kafarski, P.; Berlicki, L. Remarkable potential of the $\alpha$-aminophosphonate/phosphinate structural motif in medicinal chemistry. J. Med. Chem. 2011, 54, 5955-5980. [CrossRef]

4. Orsini, F.; Sello, G.; Sisti, M. Aminophosphonic acids and derivatives. Synthesis and biological applications. Curr. Med. Chem. 2010, 17, 264-289. [CrossRef] [PubMed]

5. Marrs, E.C.L.; Varadi, L.; Bedernjak, A.F.; Day, K.M.; Gray, M.; Jones, A.L.; Cummings, S.P.; Anderson, R.J.; Perry, J.D. Phosphonopeptides revisited, in an era of increasing antimicrobial resistance. Molecules 2020, 25, 1445. [CrossRef] [PubMed]

6. Smart, B.E. Fluorine substituent effects (on bioactivity). J. Fluor. Chem. 2001, 109, 3-11. [CrossRef]

7. Purser, S.; Moore, P.R.; Swallow, S.; Gouverneur, V. Fluorine in medicinal chemistry. Chem. Soc. Rev. 2008, 37, 320-330. [CrossRef]

8. Wanat, W.; Dziuk, B.; Kafarsky, P. New crystal structures of fluorinated $\alpha$-aminophosphonic acid analogues of phenylglycine. Struct. Chem. 2020, 31, 1197-1209. [CrossRef]

9. Cytlak, T.; Kazmierczak, M.; Skibinska, M.; Koroniak, H. Latest achievements in the preparation of fluorinated aminophosphonates and aminophosphonic acids. Phosphorus Sulfur Silicon Relat. Elem. 2017, 192, 602-620. [CrossRef]

10. Burgey, C.S.; Robinson, K.A.; Lyle, T.A.; Sanderson, P.E.J.; Lewis, S.D.; Lucas, B.J.; Krueger, J.A.; Singh, R.; Miller-Stein, C.; White, R.B.; et al. Metabolism-directed optimization of 3-aminopyrazinone acetamide thrombin inhibitors. Development of an orally bioavailable series containing P1 and P3 pyridines. J. Med. Chem. 2003, 46, 461-473. [CrossRef]

11. Qian, A.; Zheng, Y.; Wang, R.; Weie, J.; Cui, Y.; Cao, X.; Yang, Y. Design, synthesis, and structure-activity relationship studies of novel tetrazole antifungal agents with potent activity, broad antifungal spectrum and high selectivity. Bioorg. Med. Chem. Lett. 2018, 28, 344-350. [CrossRef]

12. Vitaku, E.; Smith, D.T.; Njardarson, J.T. Analysis of the structural diversity, substitution patterns, and frequency of nitrogen heterocycles among U.S. FDA Approved Pharmaceuticals. J. Med. Chem. 2014, 57, 10257-10274. [CrossRef] 
13. Nifant'ev, E.Y. Chemistry of Hydrophosphoryl Compounds; Nauka: Moscow, Russia, 1983; pp. 84-87. (In Russian)

14. Orlovskii, V.V.; Vovsi, B.A. Interaction of dialkyl phosphites with nitriles. Zhurnal Obshch. Khimii 1976, 46, 297-300. (In Russian)

15. Midrier, C.; Lantsoght, M.; Volle, J.-N.; Pirat, J.-L.; Virieux, D.; Stevens, C.V. Hydrophosphonylation of alkenes or nitriles by double radical transfer mediated by titanocene/propylene oxide. Tetrahedron Lett. 2011, 52, 6693-6696. [CrossRef]

16. Zimin, M.G.; Dvoinishnikova, T.A.; Konovalova, I.V.; Pudovik, A.N. Reaction of sodium salts of dialkylphosphorous acids with carboxylic acid nitriles. Russ. Chem. Bull. 1978, 27, 436-437. [CrossRef]

17. Rassukana, Y.V.; Kolotylo, M.V.; Synytsya, O.A.; Pirozhenko, V.V.; Onys'ko, P.P. $\alpha$-Iminotrifluoroethylphosphonates: The first representatives of $\mathrm{NH}$ imidoyl phosphonates. Synthesis 2007, 17, 2627-2630. [CrossRef]

18. Rassukana, Y.V.; Yelenich, I.P.; Synytsya, A.D.; Onys'ko, P.P. Fluorinated NH-iminophosphonates and iminocarboxylates: Novel synthons for the preparation of biorelevant $\alpha$-aminophosphonates and carboxylates. Tetrahedron 2014, 70, 2928-2937. [CrossRef]

19. Rassukana, Y.V.; Yelenich, I.P.; Bezdudny, A.V.; Mironov, V.F.; Onys'ko, P.P. Chemoselectivity of reactions of haloacetonitriles with hydrogen phosphonates: Dramatic effect of the nature of halogen atom. Tetrahedron Lett. 2014, 55, 4771-4773. [CrossRef]

20. Blanch, J.J. Determination of the Hammett substituent constants for the 2-, 3-, and 4-pyridyl and -pyridinium groups. J. Chem. Soc. B 1966, 937-939. [CrossRef]

21. Khomutnik, Y.Y.; Onys'ko, P.P.; Rassukanaya, Y.V.; Pirozhenko, V.V.; Synytsya, A.D. N-aryltrifluoroacetimidoylphosphonates. Russ. J. Gen. Chem. 2013, 83, 445-452. [CrossRef]

22. Onys'ko, P.P.; Chudakova, T.I.; Pirozhenko, V.V.; Rozhenko, A.B. $\alpha$-Ketophosphonates in the synthesis of $\alpha$-iminophosphonates. Curr. Green Chem. 2020, 7, 226-238. [CrossRef]

23. Hansch, C.; Leo, A.; Taft, R.W. A survey of Hammett substituent constants and resonance and field parameters. Chem. Rev. 1991, 91, 165-195. [CrossRef]

24. Rassukana, Y.V.; Onys'ko, P.P.; Kolotylo, M.V.; Sinitsa, A.D.; Lyzwa, P.; Mikolajczyk, M. A new strategy for asymmetric synthesis of aminophosphonic acid derivatives: The first enantioselective catalytic reduction of C-phosphorylated N-H imines. Tetrahedron Lett. 2009, 50, 288-290. [CrossRef] 